\title{
A BASELINE SURVEY OF THE GUIDING PRINCIPLES ON MANAGING FOR SUSTAINABLE DEVELOPMENT RESULTS
}

Alejandro Guerrero-Ruiz, Julia Schnatz and Chantal Verger
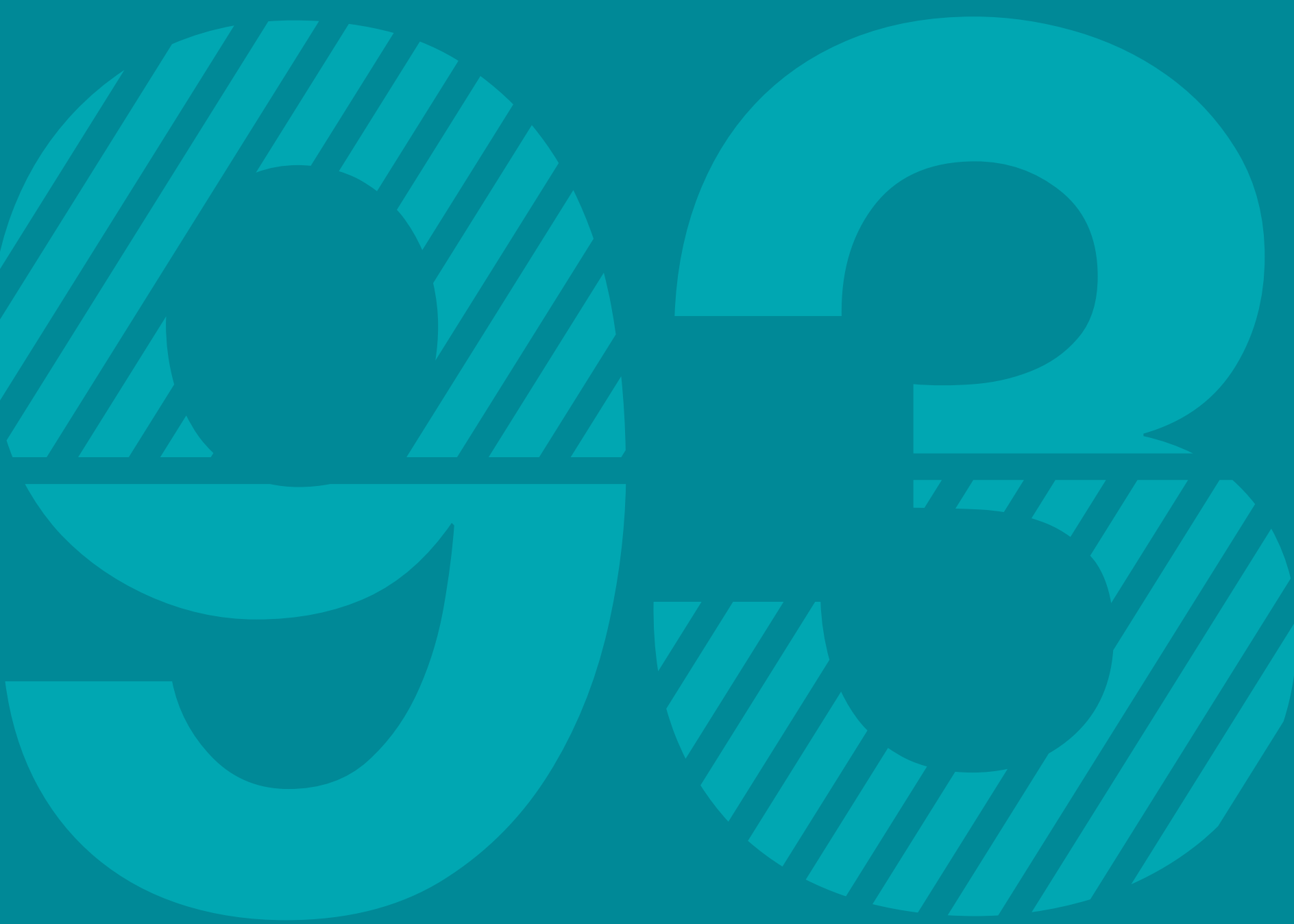

\section{OECD DEVELOPMENT CO-OPERATION WORKING PAPER 93}

Authorised for publication by Jorge Moreira da Silva, Director, Development Co-operation Directorate

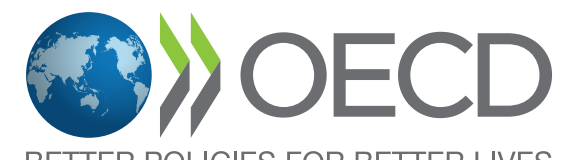





\section{OECD Working Paper}

OECD Working Papers do not represent the official views of the OECD or of its member countries. The opinions expressed and arguments employed are those of the authors. Working Papers describe preliminary results or research in progress by the authors and are published to stimulate discussion on a broad range of issues on which the OECD works. Comments on this Working Paper are welcome and may be sent to dac.results@oecd.org - Development Co-ordination Directorate, OECD, 2 rue André Pascal, 75775 Paris Cedex 16, France.

This document, as well as any data and any map included herein, are without prejudice to the status of or sovereignty over any territory, to the delimitation of international frontiers and boundaries and to the name of any territory, city or area.

Please cite this paper as Guerrero-Ruiz, A., Schnatz, J. and C. Verger (2021), "A Baseline Survey of the Guiding Principles on Managing for Sustainable Development Results", OECD Development Co-operation Working Paper no. 93, OECD Publishing, Paris. 


\section{Abstract}

This paper describes the aggregate findings of a survey conducted to assess where development cooperation providers that are members of the Results Community of the Development Assistance Committee (DAC) of the Organisation for Economic Co-operation and Development (OECD) stand with regard to the Guiding Principles on Managing for Sustainable Development Results (MfSDR) that were adopted in July 2019. Fifty bilateral and multilateral organisations participated in the survey. In addition to presenting detailed findings against each Principle, this paper also examines the main strengths and constraints providers are facing in order to align to these Principles. It also analyses some correlations between the Principles, drawing conclusions on some of the more practical consequences for systemic and tailored approaches to implementing the Guiding Principles. 


\section{Acknowledgements}

This working paper was written by Alejandro Guerrero-Ruiz, Julia Schnatz and Chantal Verger, under the strategic guidance of Rahul Malhotra, Head of the Reviews, Results, Evaluation and Development Innovation (RREDI) Division at the Development Co-operation Directorate (DCD). It was reviewed by Joëlline Bénéfice, Adviser, Centre for Tax Policy and Administration (CTP). The authors would also like to thank OECD Development Assistance Committee (DAC) Results Community Leads of the Temporary Working Groups where preliminary findings from the survey were discussed in the middle of 2020. This paper benefited from the administrative support of Ola Kasneci. It was edited by Mebrak Tareke. The authors are very grateful for all the useful comments and suggestions. Any possible error remains the exclusive responsibility of the authors. 


\section{Abbreviations and acronyms}

$\begin{array}{ll}\text { CRF } & \text { Corporate Results Framework } \\ \text { CTP } & \text { Centre for Tax Policy and Administration } \\ \text { DAC } & \text { Development Assistance Committee } \\ \text { DCD } & \text { Development Co-operation Directorate } \\ \text { GPEDC } & \text { Global Partnership for Effective Development Co-operation } \\ \text { M\&E } & \text { Monitoring and evaluation } \\ \text { MDB } & \text { Multilateral development bank } \\ \text { MfSDR } & \text { Managing for sustainable development results } \\ \text { ODA } & \text { Official development assistance } \\ \text { OECD } & \text { Organisation for Economic Co-operation and Development } \\ \text { RREDI } & \text { Reviews, Results, Evaluation and Development Innovation Division } \\ \text { SDC } & \text { Swiss Agency for Development and Co-operation } \\ \text { SDGs } & \text { Sustainable Development Goals } \\ \text { SECO } & \text { Swiss Economic Co-operation and Development Division of the State Secretariat for } \\ \text { RBM } & \text { Economic Affairs } \\ \text { UN } & \text { Results-based management } \\ & \text { United Nations }\end{array}$




\section{Table of contents}

OECD Working Paper

$\begin{array}{ll}\text { Abstract } & 4\end{array}$

Acknowledgements $\quad 5$

$\begin{array}{ll}\text { Abbreviations and acronyms } & 6\end{array}$

$\begin{array}{ll}\text { Executive summary } & 10\end{array}$

1 Background information on the baseline survey 13

What is the purpose of this baseline survey? 13

What were the steps taken to develop and administer the survey?

Who are the survey respondents? 14

\section{Providers' distance to the Guiding Principles on Managing for Sustainable}

Development Results 16

Where do providers of development co-operation stand when it comes to the Guiding Principles?

An overview

$\begin{array}{ll}\text { Key strengths and challenges } & 17\end{array}$

3 Detailed findings per Guiding Principle $\quad 21$

Guiding Principle 1: Support the Sustainable Development Goals and desired change 21

Guiding Principle 2: Adapt to context 27

Guiding Principle 3: Enhance country ownership, mutual accountability, and transparency 31

Guiding Principle 4: Maximise the use of results information for learning and decision-making 36

Guiding Principle 5: Foster a culture of results and learning $\quad 40$

Guiding Principle 6: Develop a results system that is manageable and reliable 43

4 Correlations between Guiding Principles 47

Correlations between Guiding Principle 1 and key elements of Principles 4, 5 and $6 \quad 47$

Correlations between Guiding Principle 2 and key elements of Principles 4, 5 and $6 \quad 48$

Correlations between Guiding Principle 3 and key elements of Principles 4, 5 and $6 \quad 49$

Correlations between the core components of Guiding Principles 4, 5, and $6 \quad 50$

Correlations call for holistic approaches in implementing the Guiding Principles 51 
5 Practical consequences for systemic and tailored results-based management approaches

Notes

Annex A. Survey respondents

Annex B. Survey questions $\quad 57$

Annex C. Strengths and constraints - Detailed results $\quad 59$

\section{FIGURES}

Figure 1.1. Survey respondents by category $\quad 15$

$\begin{array}{lr}\text { Figure 2.1. Overview of aggregate results } & 17\end{array}$

Figure 2.2. Range of responses regarding results-based management systems' strengths and constraints 18

Figure 2.3. Overall strengths and constraints by group 19

$\begin{array}{lr}\text { Figure 2.4. Results-based management profile clusters } & 20\end{array}$

Figure 3.1. Use of corporate results frameworks $\quad 22$

Figure 3.2. Providers that develop results frameworks mostly/systematically, by type of level 23

Figure 3.3. Extent to which results frameworks incorporate Sustainable Development Goal results indicators 24

Figure 3.4. Established methodologies are used to define the results chain at each level 25

Figure 3.5. Extent to which providers consider the current design of their organisational structure to be geared

towards achieving development results

Figure 3.6. Extent to which providers think that cross-sector approaches are promoted and included in measurement, learning, and reporting systems 27

Figure 3.7. Quality context analyses allow for a sound understanding of local dynamics and needs, and inform country- and project-level results frameworks

Figure 3.8. The conditions for success and risk factors in achieving the intended outcomes are identified and monitored during the implementation phase

Figure 3.9. Managers at the country level have sufficient flexibility to tailor their approaches and processes to each specific context (design phase)

Figure 3.10. Managers at the country level focus on achieving long-term outcomes, they are empowered to adapt a project based on changing local contexts to ensure that the expected outcomes are met (implementation phase)

Figure 3.11. Country-level results frameworks are aligned with the results frameworks of partner countries, including at the indicator level

Figure 3.12. Organisations' planning cycles at the country level are synchronised with the planning cycles of partner countries

Figure 3.13. Country and project-level results frameworks are developed in a participatory way, and involvement is sought in problem analysis, design, planning, monitoring, evaluation and in the use of results Figure 3.14. Monitoring approaches maximise the use of the monitoring and statistical systems of partner countries and their data

Figure 3.15. Efforts are made to strengthen the statistical and monitoring capacities of countries where necessary

Figure 3.16. Mutual accountability is actively pursued at the country and project-level with regular dialogue taking place among partners

Figure 3.17. Results frameworks, information and data are high quality, timely, and made publicly available

Figure 3.18. Results information from monitoring and evaluation is used for learning and decision making at all levels

Figure 3.19. Use of results information at the headquarters and the country levels

Figure 3.20. The purpose and use of results information is clearly defined among staff and partners $\quad 39$

Figure 3.21. Qualitative information is collected to complement quantitative data

Figure 3.22. Leadership communicates the purpose of results-based management and uses results information for overall programme management and to influence policy at the political level 
Figure 3.23. Leadership promotes a culture of results and learning by providing appropriate guidance, tailored incentives and resources for managing for sustainable development results

Figure 3.24. All relevant staff are trained in results-based management approaches and methods 42

Figure 3.25. There are mechanisms for peer learning on successes and failures 42

Figure 3.26. The organisation values careful experimentation in support of innovation as a way to foster better results

Figure 3.27. Monitoring and evaluation systems are adequately resourced to meet the needs of organisations 44 Figure 3.28. Monitoring and evaluation systems are set up to produce credible quantitative, qualitative data as well as evidence that meets the needs, capacities of development organisations, their partners

Figure 3.29. Results-based management is supported by adequate information systems, and it has the ability to manage quantitative and qualitative results information

Figure 3.30. The overall results-based management system is user friendly, lean and reviewed regularly to fit its purpose

Figure 4.1. Correlations between Guiding Principle 1 and key elements of Guiding Principles 4 and $6 \quad 48$

Figure 4.2. Correlations between key elements of Guiding Principle 2 and Principles 4, 5, and $6 \quad 49$

Figure 4.3. Correlations between Guiding Principles 4, 5, and $6 \quad 50$

Figure 4.4. Correlations between Guiding Principles $\quad 51$

Figure C.1. To what extent are Sustainable Development Goals indicators used in providers' results frameworks?

Figure C.2. Extent to which providers use established methodologies to develop their results frameworks (at the following levels)

Figure C.3. Results frameworks and Sustainable Development Goals indicators

\section{TABLES}

Table 1.1. Overview of the Guiding Principles

Table A.1. List of survey respondents 


\section{Executive summary}

More than ever before, development co-operation providers are called upon to join efforts and maximise the impact of individual and collective efforts to achieve the Sustainable Development Goals (SDGs). More so since the international community is now grappling with how to address a rapid response to the COVID-19 pandemic without losing sight of the long-term objectives of the 2030 Agenda.

Using results-based management (RBM) as a strategic management tool can help providers navigate complex development co-operation and humanitarian challenges reach expected results and support their sustainable development goals more effectively. That was why the Development Assistance Committee (DAC) at the OECD adopted the Guiding Principles on Managing for Sustainable Development Results (MfSDR) in July 2019. Implementing these Guiding Principles requires development co-operation providers to adapt their approaches and their systems.

The Development Co-operation Directorate (DCD) Results Secretariat conducted an online survey in late 2019-early 2020 to understand where members of the OECD/DAC Results Community stand with regard to the Guiding Principles at the time of their adoption. Fifty bilateral and multilateral organisations participated in the survey. Their responses allow us to map approaches to RBM and to establish a baseline when it comes to each guiding principle. The main findings from the survey analysis are as follows:

When it comes to developing their RBM systems, development co-operation providers are not all in the same place. The range of responses for assessing strengths and constraints is vast. This reflects discrepancies in the maturity of RBM across organisations. We can identify three distinct groups of providers. The second group can be divided into two clusters:

- Front-runners: these advanced performers have established methodologies firmly in place. They are more aligned with the SDGs, promoting cross-sector approaches. They are also decentralised enough to allow for more flexible, context-based approaches as well as adaptive management systems. Among those, are long-established bilateral providers as well as multilateral development banks (MDBs) and United Nations agencies that share well-developed approaches to RBM.

- Builders: half of the organisations are in the process of actively strengthening their RBM systems. They consider that they are making progress against most of the Principles. However, they are still struggling to mainstream a culture of results and learning across their organisations. This group is split between a small majority who think that they have a relatively good results system and others who do not feel as they have the right system in place yet. This group consists mostly of bilateral providers and a few United Nations agencies.

- Start-uppers: one in five organisations is just starting to build a culture of results and to set-up its RBM system. Most of these organisations have a limited presence in partner countries. Most of them have focused on results at a strategic level, but they fail to adapt to the context. They are also still struggling to enhance country ownership and to make good use of results information.

Organisations rank themselves higher on strategy than on their ability to implement RBM systems. While the overall degree of alignment of each provider varies across principles, half of the providers consider their current organisational set-up for implementing RBM to be inadequate. Overall, they assess themselves 
as doing better in terms of aligning to Principle 2 (adapting to context) and Principle 4 (maximising the use of results information) and, to a lesser extent to Principle 1 (supporting sustainable development goals and the desired change) as well as to Principle 3 (enhancing ownership, mutual accountability and transparency) but alignment lags behind with regard to Principle 5 (fostering a culture of results and learning) and Principle 6 (having a manageable and reliable results system). Responses to each Guiding Principle show a more nuanced picture:

Guiding Principle 1: Strategic vision: the vast majority of providers have or are developing results frameworks using established methodologies, though more so at the project level. Apart from United Nations agencies, most providers still only make limited use of the SDG indicators in their results frameworks. However, those with a corporate results framework in place are making efforts to align these frameworks with the SDGs. With the exception of the MDBs, most providers are not promoting cross-sector approaches.

Guiding Principle 2: Adapt: most providers feel that they are already performing well in terms of conducting quality context analysis, identifying and monitoring the conditions for success and risk factors, as well as empowering staff to adapt and tailor their approaches and processes to each specific context. However, there are limits to this since having flexibility does not necessarily mean it will work in practice.

Guiding Principle 3: Effectiveness: almost half of the providers align their country level results framework with those of partner countries and encourage synchronising programming cycles. More than half of providers develop their results frameworks in a participatory way. However, two thirds do not optimise the use of partner countries' monitoring and statistical systems, even though they provide support to strengthen these systems in at least some cases. While two-thirds of providers state that they actively pursue mutual accountability, they also recognise that their provision of quality and timely results information remains limited.

Guiding Principle 4: Use: for many donors, ensuring that results data are used for learning and decision making is a challenge. They use results information at the country level more frequently than at the headquarters level. Using results information for learning comes last for all types of providers, both at the headquarters and country level. While a majority of providers ensure that the purpose and use of results data and information are made clear, they recognise that more clarity is needed on what drives the collection and use of qualitative information.

Guiding Principle 5: Culture: for the majority of providers, leadership does not fulfil its role of fostering a culture of results, and most providers feel that there is a lack of appropriate guidance and tools. Promoting experimental innovation is not yet a common practice. However, training in approaches to RBM tends to increase among more-established organisations. In addition, half of the providers offer opportunities for peer learning on successes and failures.

Guiding Principle 6: Systems: the majority of providers think that their monitoring and evaluation (M\&E) systems produce credible quantitative and qualitative evidence that meet their needs. However, they also feel as though they are not adequately resourced. The majority of them also feel that they lack adequate information systems, that their RBM system is not user-friendly, and that it is not reviewed regularly enough to meet its purpose.

Strong interdependences exist between key elements of the Guiding Principles. The use of results information for learning and decision making at all levels depends on all the other principles. In particular, this is strongly correlated with the prevalence of results frameworks and cross-sector approaches as well as the quality and transparency of results information. It also depends on the extent to which leadership promotes an internal culture of results and learning and the level of authority that is given to managers in the field. 
Overall, these findings call for strategic approaches to be matched by adequate implementation measures. In particular:

- Enhancing and sustaining a broad culture of results and learning is a pre-requisite: leadership has a key role to play in promoting a culture of results and learning by communicating the purpose of RBM, leading by example in terms of using results information, as well as providing RBM guidance, training and resources while enhancing the scope for peer learning within the organisation. Leadership is also responsible for setting up an appropriate incentive structure, which is one of the key constraints indicated by most providers.

- It is also essential to have adequate monitoring, evaluation and information systems in place in order to collect, process, and share data. If these are built in ways that allow users to review their results data, as well as seeing where their data feeds into the whole organisation, the systems will give both users and producers an even greater incentive to actually collect and use the data.

- Approaches need to be tailored to each institutional context as well as how mature RBM systems are. Taking a holistic approach can help the organisations that are bolstering their RBM systems to take into account correlations between the guiding principles, building on synergies and managing tensions that exist between key elements of these principles. Those who are building different components of their systems will benefit from iterative approaches, where taking stock regularly could lead to lessons learned and best practices to incrementally improve the system. Frontrunners can lead in developing innovative approaches to address complexity and unpredictability that will be useful to the overall community.

- Using the SDGs can help resolve tensions. Using the SDGs as a common results framework can help to reconcile corporate results frameworks (CRF) with standardised indicators that can be aggregated for accountability purposes, and country-level results frameworks with context-based indicators to facilitate alignment to national policies and frameworks. The SDGs can also help organisations move towards more coherent, cross-sector and interdisciplinary approaches, which could then be incorporated into measurement, learning and reporting systems. 


\section{Background information on the baseline survey}

\section{What is the purpose of this baseline survey?}

The Development Assistance Committee (DAC) approved new Guiding Principles on Managing for Sustainable Development Results in July 2019 (OECD, 2019 ${ }_{[1]}$ ). This marks an important step towards recognising the importance of the results agenda as a catalyst for more effective development co-operation. The Principles reflect a changing context for development co-operation as well as a broader set of actors. Their purpose is to guide development organisations as they set up or fine-tune RBM approaches that are fit for the 2030 Agenda for Sustainable Development. The Guiding Principles will also help support institutional change as well as promoting a culture of results.

Implementing the Guiding Principles on MfSDR require development co-operation providers and their partners to adapt their approaches and their systems. Yet, the need for policy reform and behavioural change depends on each organisation's institutional context and how mature their RBM systems are.

In order to understand fully where members of the OECD/DAC Results Community stand with regard to the Guiding Principles at the time of their adoption, the Results Secretariat conducted an online survey between November 2019 and May 2020. Its specific objectives were to:

1. Establish a broad baseline, using aggregate information to identify where members are making progress or lagging behind, and their overall strengths and constraints in aligning with the principles. This would make it easier to assess the overall progress over a longer period of time. ${ }^{1}$

2. Map the RBM approaches of the members in terms of their consequences for the capacity and scope for each member to collect, measure and use the results information at each level of the development co-operation programme. This mapping will be used in conjunction with findings from research conducted by the Results Secretariat on how to make better use of the SDGs as a common framework for results, with a view to provide members with tailored guidance and tools that will be developed to help implement the Guiding Principles.

This paper presents the aggregate findings of the online survey. Chapter one presents the purpose and methodology of the survey as well as the different categories of the respondents. Chapter two analyses the providers' distance to the Guiding Principles on MfSDR. It explores the main strengths and constraints identified by those who responded to the survey in as far as that relates to the Principles, and outlines emerging RBM profiles. Chapter three presents more detailed findings against each Principle. Chapter four takes a step back and looks at the correlations between the Principles, drawing conclusions on some of the more practical consequences for systemic and tailored approaches to implementing the Guiding Principles. Unless specified otherwise, all tables and figures are based on responses from the survey, and no source has been cited unless it came from somewhere other than the survey. 


\section{What were the steps taken to develop and administer the survey?}

The survey was developed by the Results Secretariat in October 2019. Its structure is aligned to the Guiding Principles, with questions focusing on the key elements of each principle. The survey concludes with a question on the overall strengths and constraints of the development co-operation provider's ability to apply the Guiding Principles (see the survey questions in Annex B).

The survey was tested and launched at the end of October 2019. By the initial deadline of 27 November 2019, 30 institutions had responded to the survey. The Results Secretariat presented a snapshot of aggregate responses at the December 2019 workshop of the OECD/DAC Results Community.

The deadline was then extended to early May 2020 in order to give more organisations the opportunity to respond.

\section{Who are the survey respondents?}

The survey targeted members of the OECD/DAC Results Community. Ultimately, 50 official development co-operation institutions participated, including all but one DAC member as well as a number of multilateral providers of development co-operation (see the list of respondents in Annex A).

In addition to presenting aggregated responses, disaggregating the responses by "donor type" allows for a more nuanced analysis, accounting for different organisational and institutional realities across providers. In particular, recognising that bilateral providers of development co-operation are quite diverse, they are split in two sub-groups depending on when they started reporting their official development assistance (ODA) to the OECD. This criterion is used as a proxy for institutional capacity developed over time, as reporting ODA requires members to develop policies, processes, systems and dedicated resources and staffing so as to track and report accurate information on their development co-operation activities. Four categories of respondents were identified:

i) Bilateral providers/Group A (who started reporting ODA statistics to the OECD before 2010);

ii) Bilateral providers/Group B (others);

iii) Multilateral development banks (MDBs); and

iv) United Nations (UN) agencies.

Figure 1.1 below presents the number of respondents per category, taking into account that in the case of five countries of bilateral providers/Group A, the survey was completed by different institutions in charge of development co-operation. 
Figure 1.1. Survey respondents by category

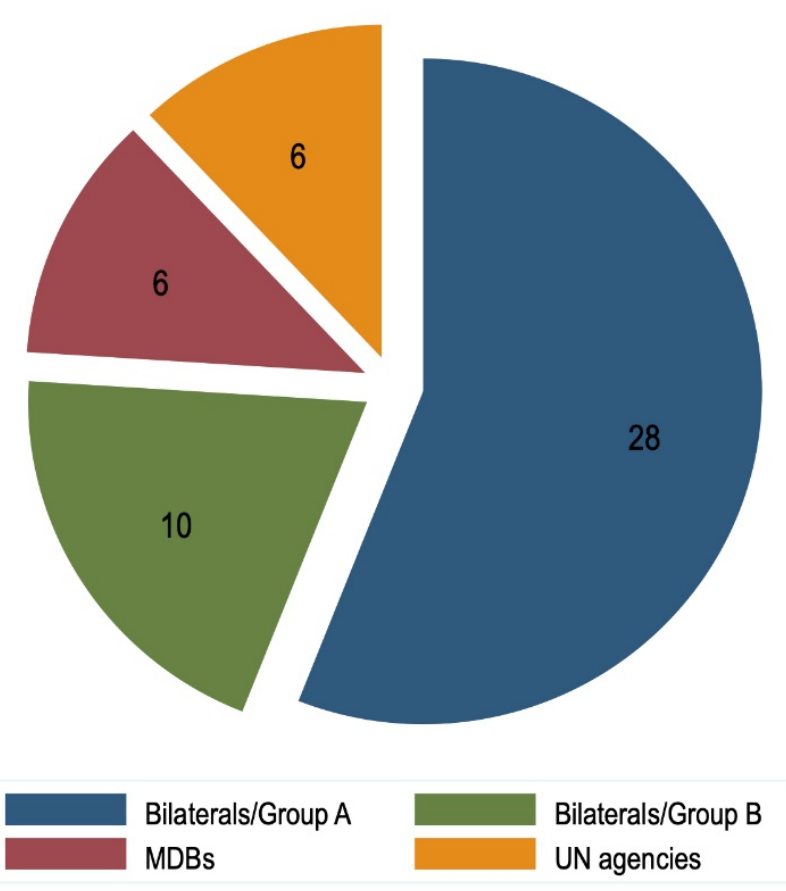

Table 1.1. Overview of the Guiding Principles

\begin{tabular}{l|l}
\hline $\begin{array}{l}\text { Guiding Principle 1: Support sustainable development goals and desired } \\
\text { change }\end{array}$ & $\begin{array}{l}\text { Managing for Sustainable Development Results (MfSDR) approaches } \\
\text { should maximise the impact of efforts towards achieving social, } \\
\text { economic and environmentally sustainable development that leaves no } \\
\text { one behind. }\end{array}$ \\
\hline $\begin{array}{l}\text { Guiding Principle 2: Adapt to context } \\
\begin{array}{l}\text { Guiding Principle 3: Enhance country ownership, mutual accountability } \\
\text { tailoring to different operational contexts, modalities of engagement, and } \\
\text { types of partnerships. }\end{array}\end{array}$ & $\begin{array}{l}\text { MfSDR approaches should foster development effectiveness by } \\
\text { strengthening and using partner countries' systems for strategic } \\
\text { planning, monitoring and statistics, and promoting participatory } \\
\text { approaches to enhance ownership, mutual accountability and } \\
\text { transparency. }\end{array}$ \\
\hline $\begin{array}{l}\text { Guiding Principle 4: Maximise the use of results information for learning } \\
\text { and decision-making }\end{array}$ & $\begin{array}{l}\text { While responding to communication and accountability needs, results } \\
\text { information should systematically be used for learning and decision- } \\
\text { making in order to improve delivery and enhance impact. }\end{array}$ \\
\hline $\begin{array}{l}\text { Guiding Principle 5: Foster a culture of results and learning } \\
\text { Guiding Principle 6: Develop a results system that is manageable and } \\
\text { reliable }\end{array}$ & $\begin{array}{l}\text { A culture of results should be promoted and sustained through consistent } \\
\text { leadership, and supported by appropriate guidance, tools and capacity } \\
\text { building as well as proper incentives. }\end{array}$ \\
\hline $\begin{array}{l}\text { While providing credible results information that can be used by all } \\
\text { partners, results frameworks, measurement and reporting systems need } \\
\text { to be suitable to each organisation's needs and capacities. }\end{array}$
\end{tabular}

Note: The full text of the Guiding Principles can be found under the reference OECD, $\left(2019_{[1]}\right)$, Managing for Sustainable Development Results: Guiding Principles, https://dx.doi.org/10.1787/44a288bc-en 


\section{Providers' distance to the Guiding Principles on Managing for Sustainable Development Results}

When it comes to developing their results-based management (RBM) systems, not all providers are at the same stage. They also have different governing structures and operating models, which impacts their approach to RBM. These differences transpire through a range of strengths and constraints in terms of aligning to the Guiding Principles and transforming development agencies into more results-oriented, effective organisations. While the Principles provide a framework that could apply to all development organisations, regardless of their scope or their degree of maturity, there is no perfect model by which a provider would fully align to every Principle. Different providers have different needs, and each organisation can implement the Principles in accordance with its mandate and its complexities. Identifying different provider profiles could help tailor support and guidance to each institutional context.

This chapter draws on responses to the survey, in order to give an overview of where providers of development co-operation stand with regard to each Guiding Principle, based on their own perceptions. This chapter then analyses what respondents consider to be their organisation's main strengths and constraints in terms of how those align with the Principles. It then explores the Principles' core components and the synergies or tensions that providers of development co-operation need to be able to manage. Finally, it outlines the RBM profiles that emerge from an analysis of responses to the survey, concluding with some practical approaches for how providers with different profiles could align with the Principles.

\section{Where do providers of development co-operation stand when it comes to the Guiding Principles? An overview}

The survey offers a baseline of where development co-operation practitioners stand vis-à-vis the Guiding Principles at the time of their adoption. On a scale of one (no/marginally) to four (systematically) the survey questions ${ }^{2}$ asked about the key components of each Guiding Principle, using the same structure throughout the process. The average score per Principle indicates how the organisations assess their alignment to each Guiding Principle.

The overall degree of alignment of each provider varies greatly from one Principle to another. Figure 2.1 illustrates the relative positions for each Guiding Principle based on average weighted scores for sets of related survey responses, showing a huge discrepancy between each Guiding Principle.

Adapting to context (Guiding Principle 2) and maximising the use of results information for decisionmaking and learning (Guiding Principle 4) appear as the two Principles where most providers consider they are already performing well. When the process of developing the Guiding Principles was launched in 2018, many providers were driven by the need to reconcile results-based management approaches with adaptive management and to make better use of results information for decision-making and learning, going beyond accountability and communication purposes. Being exposed to these matters 
throughout the process might have helped providers perform well under these two related Guiding Principles. However, chapter two reveals a distinction between policy and practice in terms of how respondents commented in the survey, which needs to be explored further.

Figure 2.1. Overview of aggregate results

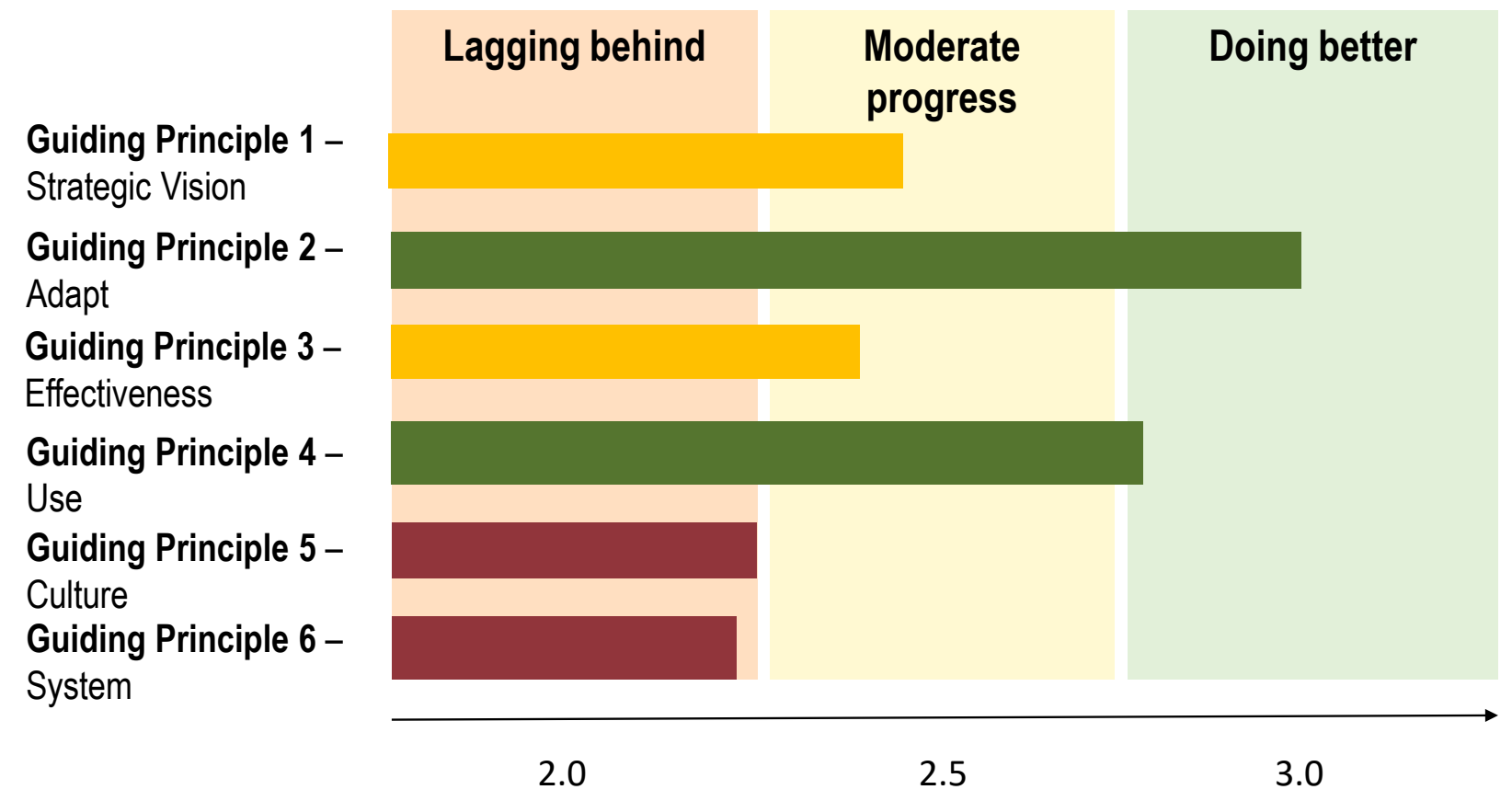

Note: Authors' assessment of relative positions for each Guiding Principle based on average weighted scores for sets of related survey responses.

In contrast, most providers are comparatively lagging behind in building a culture of results (Guiding Principle 5) and having a manageable and reliable results system (Guiding Principle 6). These two Principles support all the other principles. Building a culture of results cuts across the different administrative layers of an organisation, involving staff from top management to programme officers and evaluators and often requires coherent change management processes. Likewise, having in place a manageable and reliable results system is a pre-requisite for being able to focus on results. Given their overall weak performance so far, in future, development co-operation providers will need to pay particular attention to these two principles.

When it comes to supporting the SDGs and the desired change (Guiding Principle 1) and enhancing ownership, mutual accountability and transparency (Guiding Principle 3), performance has been moderate. These Principles not only define the vision. They also articulate how providers can ensure their results aproaches and systems are geared towards achieving sustainable development results and enhancing development effectiveness. With such moderate performance, providers need to pay more attention to the key elements of both Principles.

\section{Key strengths and challenges}

Improving the design of organisational structures and processes to steer providers towards sustainable results management requires constant fine-tuning. Similar processes are also taking place within partner countries. Respondents were asked to rank themselves on their strengths and constraints in order for them to develop their RBM system on a scale of one (huge weakness) to ten (huge strength). Ten different 
categories were assessed, ranging from strategic considerations on the existence of policies in place that enable RBM to finding appropriate IT systems and incentives to support the implementation of RBM approaches.

The range of responses for assessing strengths and constraints is vast, reflecting discrepancies in the maturity of RBM across organisations. This discrepancy is particularly important when it comes to assessing the IT systems required to support RBM (Figure 2.2).

Organisations rank themselves higher on strategy than on their ability to implement RBM systems. In particular, policies and the formal demand for results seem to be strong across the board, while IT systems and the institutional incentive structure seem to be lacking among respondents. This suggests there is commitment among leadership and a clear mandate in support of implementing RBM systems. It also echoes the fact that the results culture (Principle 5) and results systems (Principle 6) are seen as two Principles that are lagging behind all the other Principles (see Figure 2.2), due to a lack of incentives and adequate IT systems. Overall, this calls for strategic approaches to be matched by adequate implementation measures.

Figure 2.2. Range of responses regarding results-based management systems' strengths and constraints

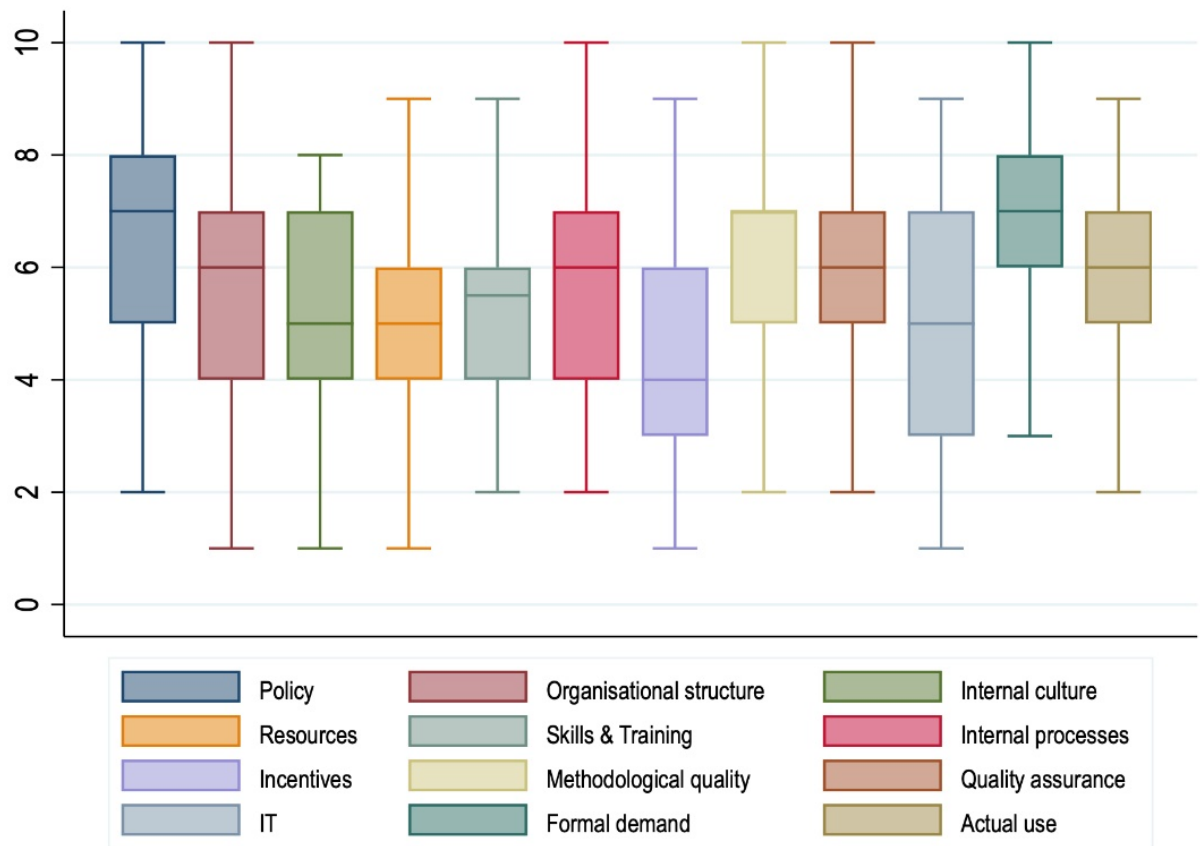

Half of the providers (25 out of the 50 surveyed organisations) consider that their current organisational set-up does not (yet) fully implement RBM to its full potential (Table C.1 in Annex C). These providers report substantive progress in establishing policies that enable RBM, thereby creating a formal demand for results information. They also report on the existence of processes and quality assurance mechanisms that make it possible to generate and make practical use of results information, particularly for accountability and communication purposes, although the latter are not yet considered "good enough". They share a similar need to continue improving RBM skills as well as shaping the organisational culture alongside other more results-oriented providers. In contrast, they identify weaknesses in terms of an organisational structure which supports RBM, with roles, responsibilities and governance around results work not clearly defined, inadequate IT systems that are more geared towards financial information than results data, as well as a comparative lack of dedicated resources and incentives for good RBM practices. 
MDBs and UN agencies report organisational set-ups and systems that are supportive of RBM more frequently. Some $80 \%$ of MDBs and $85 \%$ of UN agencies consider that their organisational set-up is geared largely towards results - although only half consider this to be a feature that is applied systematically. This was made possible by supportive policies and a strong demand for results information as well as adequate resources and appropriate organisational structures and tools such as quality assurance, methodologies, and internal processes. Still, these organisations need to pay more attention to creating incentives, an internal culture of results, staff skills and training, as well as their IT systems (Figure 2.3).

Just over half of bilateral providers report organisational designs that are supportive of RBM. This is less common ( $9 \%$ ) among recently established bilateral providers (Group B), since the internal demand and the process of institutional development for RBM takes time to consolidate. Long-established providers (Group A) tend to follow the same pattern as multilateral organisations, with their strengths lying in supportive policies, an existing formal demand for results-information and adequate organisational structures and tools. Similarly, they need to strengthen the skills and training of their staff, enhance an internal culture of results as well as updating their IT systems. In addition, many bilateral providers mention the need for more resources to support the RBM approach.

The incentive structure is the biggest weakness of all respondents, except for recently established bilateral providers, where dedicated resources score even lower. Policies and formal demand for results information reporting seem to be closely related, ranking highest across all four groups.

Figure 2.3. Overall strengths and constraints by group

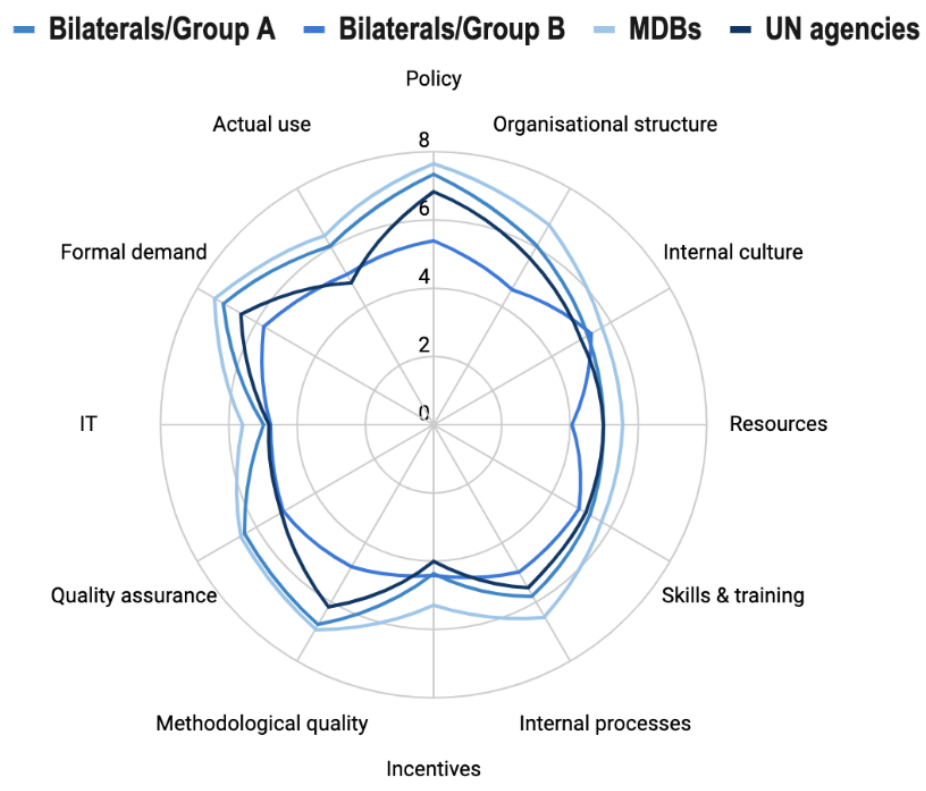

\section{Emerging results-based management profiles}

The vast majority of providers $(80 \%)$ follow the average profile illustrated in Figure 2.1: they asses themselves as performing well against Guiding Principles 2 and 4, moderately against Principles 1 and 3 , and weakly against Principle 5 (Figure 2.4). Meanwhile, the level of overall performance varies a lot within them (as does the range of responses assessing their strengths and constraints). Responses to the survey therefore allow us to identify three different profiles that share common characteristics in their approach to RBM as seen by their level of alignment to each Guiding Principle: "front runners", "builders", and "startuppers". ${ }^{3}$ 
Front-runners score highest on Guiding Principles 1,2 and 4 but assess themselves as performing moderately when implementing Principles 3,5 and 6 (cluster 1 in Figure 2.4). These organisations tend to have established methodologies firmly in place, aligning more closely with the SDGs, promoting cross-sector approaches, as well as being decentralised enough to allow for context-based approaches and adaptive management systems. Among these 16 organisations are long-established bilateral providers as well as MDBs and UN agencies that share well-developed approaches to RBM. Despite these strengths, only a few of the organisations within this group are performing well against Principles 4 and 5 , and nearly all are still struggling to set up a manageable and reliable RBM system (Principle 6).

Builders follow the pattern at a medium level, performing well against Guiding Principles 2 and 4, moderately against Principles 1 and 3, and weakly against Principle 5. There are 25 organisations in this profile, made of bilateral organisation and a few UN agencies that are strengthening their RBM system. Within them, 15 organisations assess themselves as performing well relatively against Principle 6 (cluster 2 in Figure 2.4) and 10 organisations as performing relatively badly against this Principle 6 (cluster 3 in Figure 2.4) either because they are only just beginning to put in place their RBM systems or because they are more self-critical.

Start-uppers are performing evenly across the Guiding Principles, but they are still far from aligning with them. Among these 9 organisations, some have focused on results at a strategic level (Guiding Principle 1) but they fail to adapt to context and almost all of them struggle to align to the Busan Partnership Agreement for effective development co-operation (Guiding Principles 2 and 3). Most of them are not able to make good use of results information and they are only starting to build an internal culture of results and learning (Guiding Principles 4 and 5). Apart from a few exceptions, these providers see their ability to collect and process data across the organisation as inadequate (Guiding Principle 6). These organisations are starting to set-up their RBM system with a limited presence in partner countries (cluster 4 in Figure 2.4).

Finally, one organisation in Figure 2.4 stands out. This development organisation with a solid RBM approach performs well against all Guiding Principles except for Principles 2 and 3, as it is centralised, which affects flexibility and transparency when it comes to RBM. While only one survey participant belongs to this categorisation, it belongs to a type of development organisation and funds that have been increasing in number within the bilateral and multilateral ecosystem in recent times.

\section{Figure 2.4. Results-based management profile clusters}

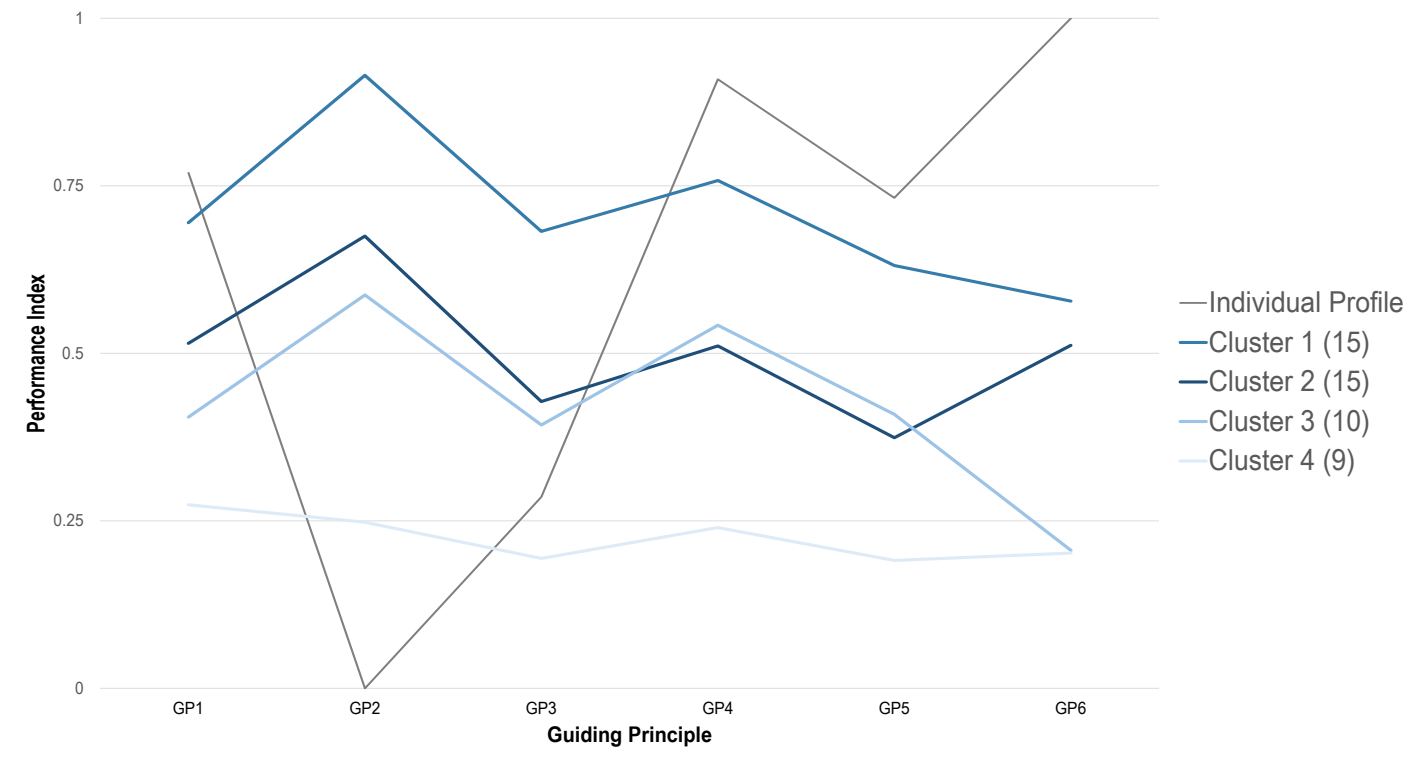

Note: Thickness of lines and number in brackets in the legend indicate the number of providers each cluster contains. 


\title{
3 Detailed findings per Guiding Principle
}

\author{
Guiding Principle 1: Support the Sustainable Development Goals and desired \\ change
}

This Guiding Principle lays the foundation for managing results into the 2030 Agenda era. Its first key element calls on members to ensure their results systems are linked inherently to the ultimate goal of attaining global or national development frameworks. This includes the 2030 Agenda for Sustainable Development with the SDGs and the pledge to leave no one behind, as well as the Paris Agreement on Climate Change.

To put this into practice, the second key element in the Guiding Principles invites development organisations to anticipate the results they expect. Along with indicators that can be measured against the SDGs, and to design and apply agile methodologies at the corporate, portfolio, country, sector, programme and project levels in order to achieve these results. Broadly speaking, Corporate Results Frameworks (CRF) are defined as instruments. They can provide overall strategic direction to development co-operation programmes as well as results information at the corporate level. At the same time being appropriate for initiating alignment with the SDGs.

\section{Setting up results frameworks}

The survey shows that the vast majority of providers have $(56 \%)$ or are developing $(30 \%)$ corporate results frameworks. This is already the norm among multilateral providers, and half of the providers established before 2010 already have an overall results framework in place (Group A). More recently established providers rely on this type of overarching results frameworks at a lower rate (20\%) (Group B) while $60 \%$ are currently in the process of developing them (Figure 3.1). 
Figure 3.1. Use of corporate results frameworks
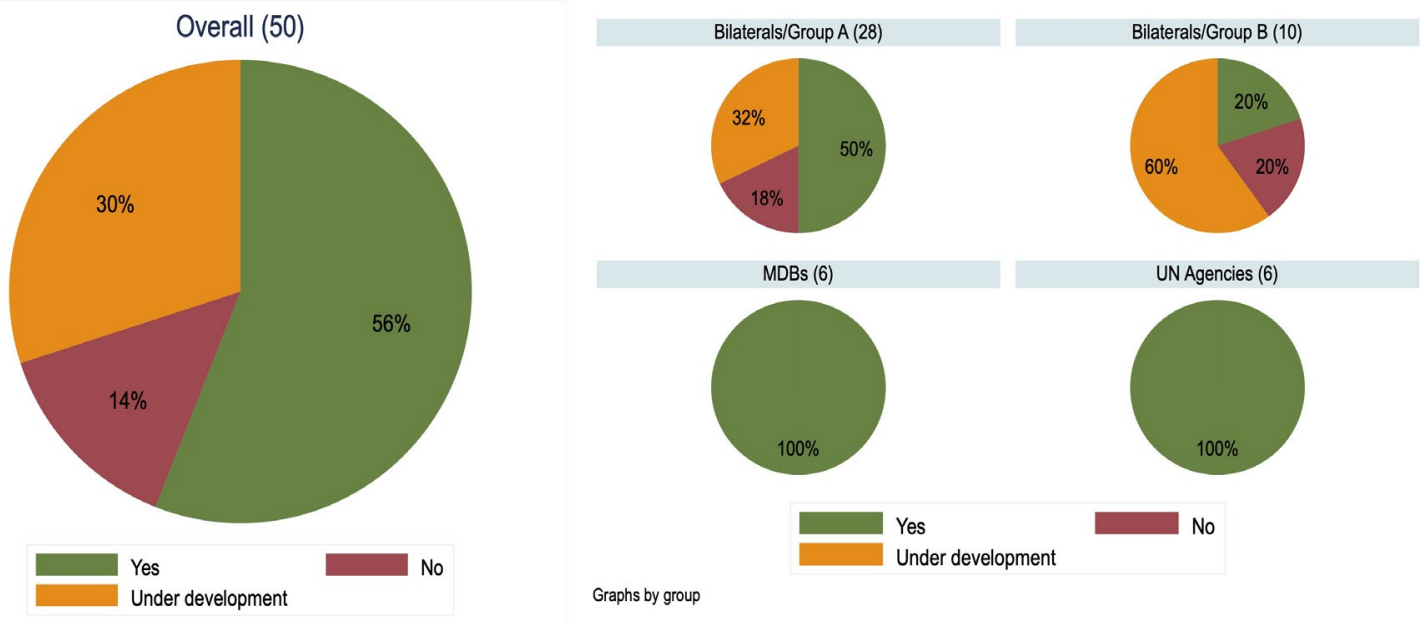

The most common results frameworks are established at the project level. Almost all providers develop these frameworks at the project level. This allows them to monitor the progress of their activities (Figure 3.2).

Almost all multilateral organisations develop results frameworks at the country level, a practice that is increasingly being shared by bilateral providers. Country results frameworks provide strategic guidance to the development co-operation programmes supported by a provider in a particular country. The results information is essentially a balancing act between a project/programme results framework and the CRF. Out of 50 bilateral and multilateral providers, about three-quarters use country-level results frameworks, and 25 providers report using them in most (if not all) of their partner countries. This is common practice for MDBs, UN agencies and most of the long-established bilateral donors. Nevertheless, a limited number of bilateral providers do not operate with specific country results frameworks. Instead, they provide inputs towards developing the results frameworks of their implementing partners. Meanwhile, most of the newly established bilateral providers have not developed results frameworks at the country level.

Results frameworks at the sector or thematic level are developed less frequently, in particular by bilateral providers. This reflects the fact that most of them have not yet adopted sector wide or thematic approaches. In a context where a decreasing level of ODA is programmed in country, providers will need to pay more attention to develop sector or thematic results framework. This is to ensure clear objectives, targets and indicators that are set to measure the results of their support which is programmed and managed at a corporate level. 


\section{Figure 3.2. Providers that develop results frameworks mostly/systematically, by type of level}

Graph shows percentage of respondents indicating this happens systematically or in the majority of cases.

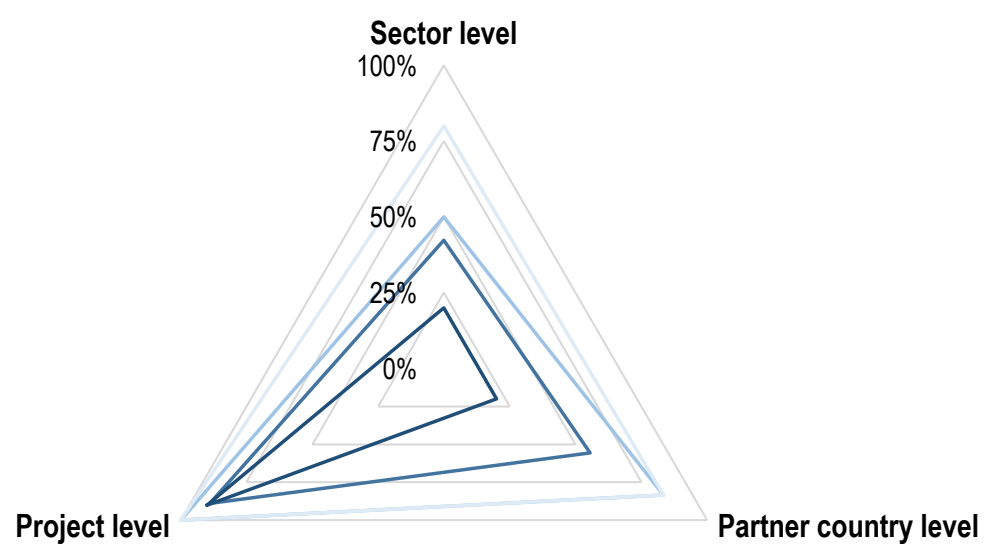

Group A - Group B - MDBs UN agencies

\section{Aligning results frameworks to the SDGs}

Overall, providers still make limited use of the SDG indicators in their results frameworks. This is particularly the case at the project level where a large number of providers do not use them systematically or in the majority of cases. The UN agencies are those making more consistent use of the SDG indicators in their results frameworks, which comes as no surprise (Figure 3.3).

Most providers with a CRF report making efforts to align the CRF to the SDGs. For many providers, what has been challenging is how to best develop or improve CRFs so that they can be aligned to the SDGs. The UN agencies align their CRFs to the SDGs systematically or in the majority of cases; and most of the others who are currently developing a CRF for their entire co-operation programme report a desire to align to the SDGs. As reported in Aligning to the SDGs: Stepping up action (OECD, forthcoming $\left.{ }_{[2]}\right)$, such alignment happens in 15 out of 27 existing CRFs - although this is done by relying on official SDG indicators, derived indicators or proxy indicators to varying degrees. A handful of providers have achieved very high levels of SDG alignment in their most recent CRF. (See more details per categories of donors in Figure C.1 in Annex $\mathrm{C})$.

At the country level, alignment is limited for most providers apart from UN agencies. A number of bilateral and multilateral providers report difficulties using SDG indicators extensively at this level. In a number of cases, this reflects tensions between various levels of alignment (to CRFs, partner country results framework and the SDGs). Research shows that a number of technical, organisational and political obstacles are also at stake. (OECD, forthcoming[2])

Overall, at the project level, SDG indicators are used the least by providers in their results frameworks. Across all categories, less than $50 \%$ of providers have stated that SDG indicators are used at least in most cases, except for UN agencies where they are used in the majority of cases if not systematically, at an average rate of $60 \%$. 
Figure 3.3. Extent to which results frameworks incorporate Sustainable Development Goal results indicators

Graph shows percentage of respondents indicating this happens systematically or in the majority of cases.

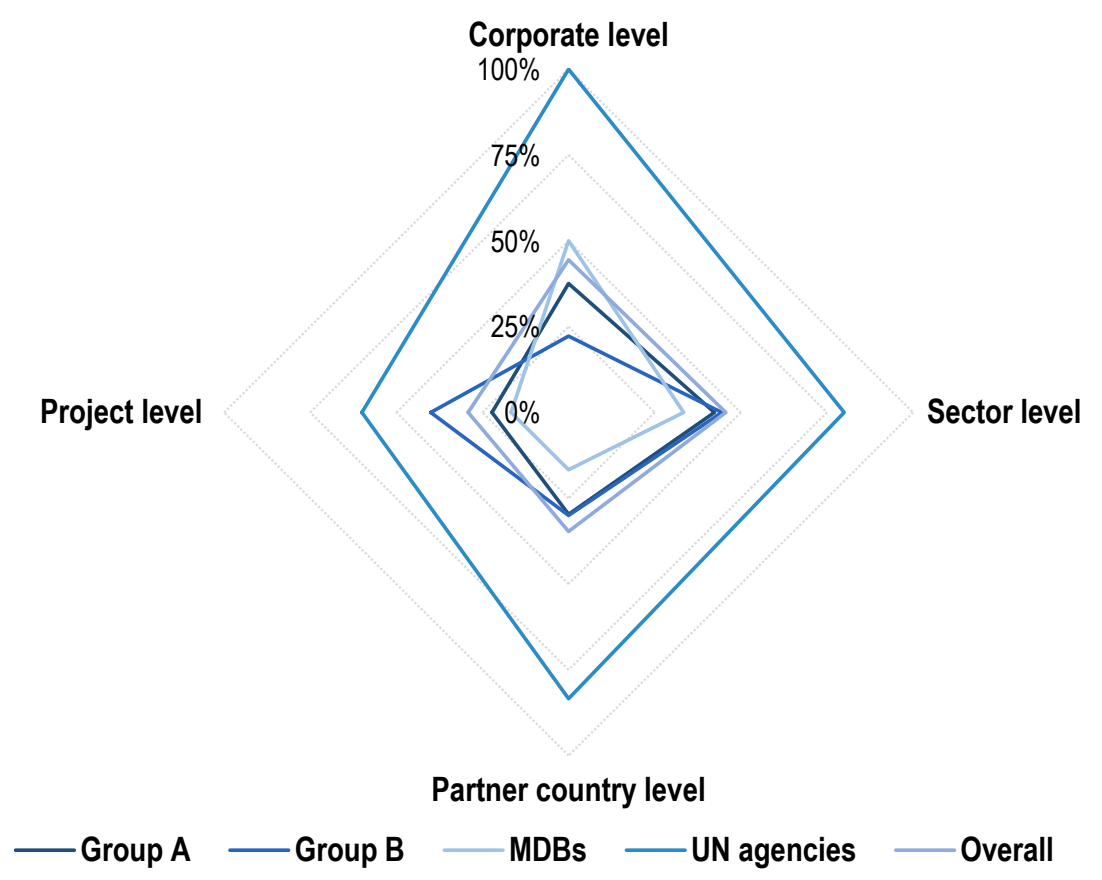

\section{Using established methodologies to define results chains}

Established methodologies to develop results frameworks are most widely used at the project level across all categories of providers, spearheaded by the UN agencies; all of them use methodologies such as theories of change and logical frameworks, and in some cases, this happens systematically. Overall, $88 \%$ of providers report using such methodologies at the project level, while at the corporate level only $39 \%$ of all respondents are using them in the majority of cases or systematically. In contrast, new bilateral providers (Group B) do not seem to use established methodologies to develop their results frameworks. It is only at the project level that $30 \%$ of them use these methodologies some of the time or most of the time (Figure 3.4).

Clearly, the gap between using established methodologies at the project and corporate levels is a reflection of how, for a long time, funding models for project- and programme-based work have made it mandatory to link an input to a deliverable through a results chain that relies on a methodology. At the corporate level, this becomes more complex, and for a long time it was considered less mandatory to apply such a methodology. This requires a strategic vision as well as a solid organisational structure and culture, which only a few providers have in place. It is also worth noting that the use of such methodologies may vary from one provider to another, ranging from a rigid and linear way to creating 'living documents' that change over time in accordance with the context. 
Figure 3.4. Established methodologies are used to define the results chain at each level

Graph shows percentage of respondents indicating that this happens systematically or in the majority of cases.

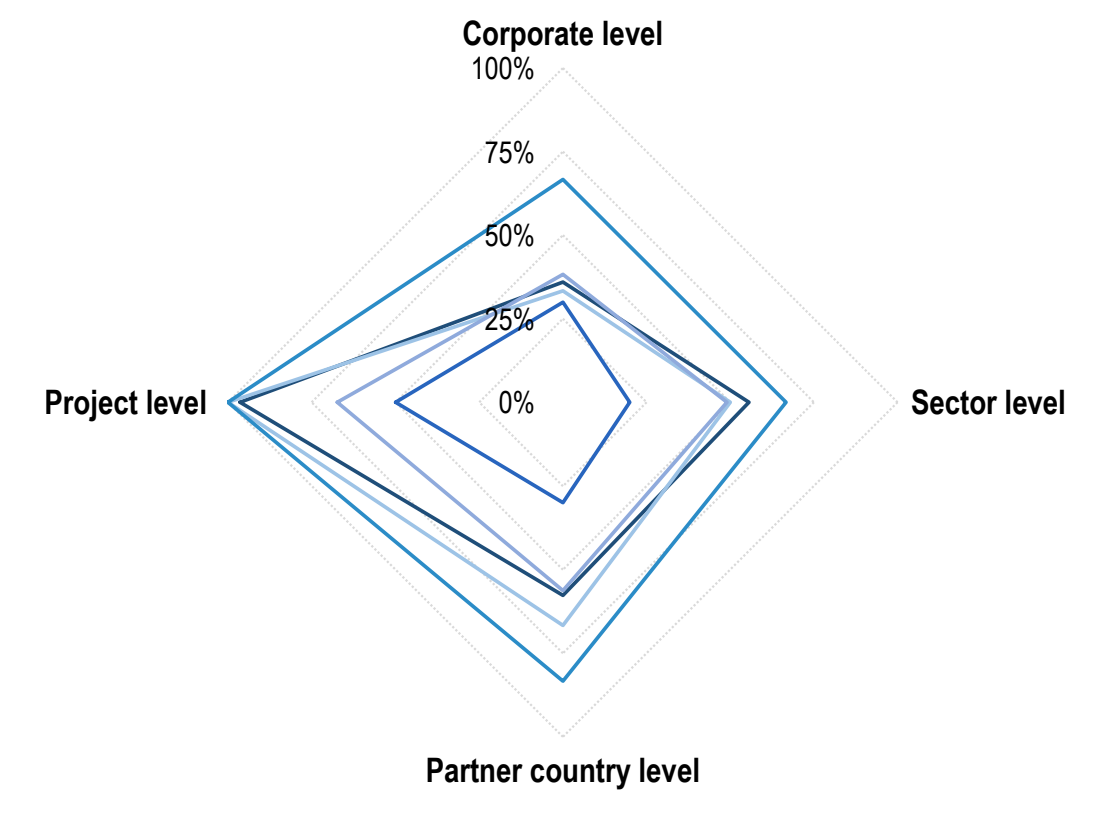

- Group A - Group B - MDBs - UN agencies - Overall

\section{Organisational structure that is geared towards development results}

Multilateral organisations assessed themselves as well-equipped to deliver development results. More than $80 \%$ of multilateral organisations said the current design of their organisational structure was geared towards achieving development results systematically or in the majority of cases. In contrast, this is only the case for $54 \%$ of long-established bilateral providers and $10 \%$ of newly established-providers. While the latter are still establishing their results systems, the governing models of the MDBs and UN agencies, and their need to be accountable, explains why these bodies have engaged in managing for results earlier and in a more structured way than bilateral providers (Figure 3.5). 
Figure 3.5. Extent to which providers consider the current design of their organisational structure to be geared towards achieving development results

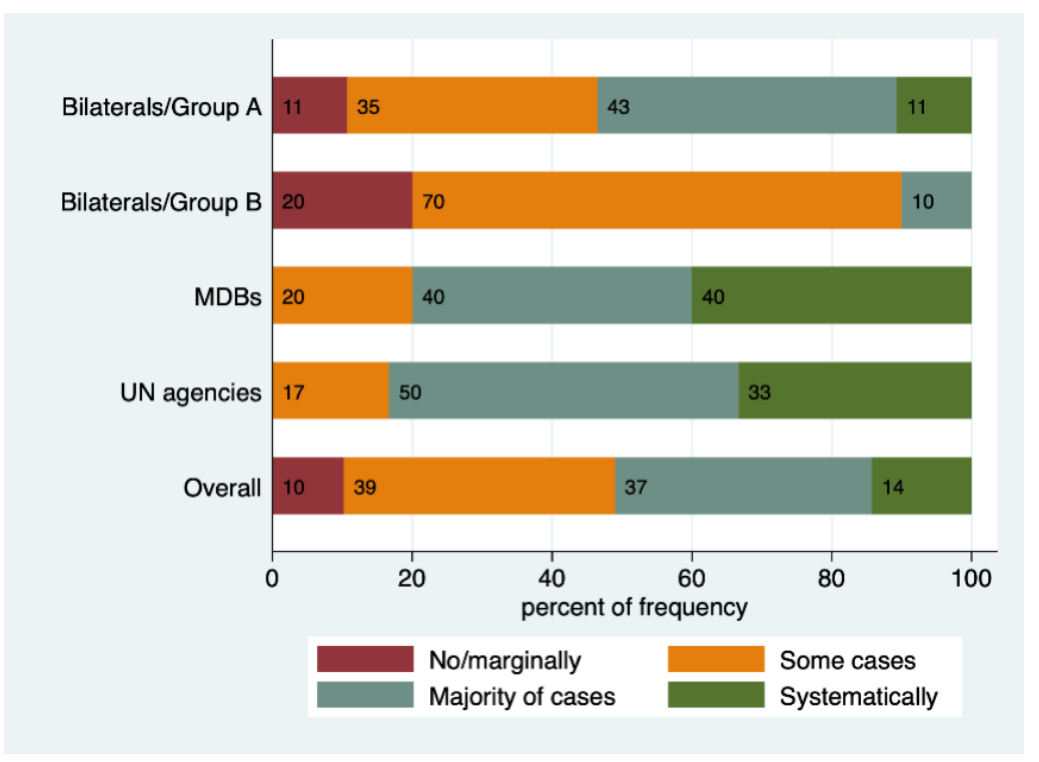

\section{Promotion of cross-sector approaches}

With the exception of the MDBs, most providers are not promoting cross-sector approaches. Two thirds of the MDBs consider that cross-sector approaches are promoted and included in measurement, learning and reporting systems systematically or in the majority of cases (Figure 3.6). This figure drops to $50 \%$ for UN agencies and it drops to $37 \%$ for long-established bilateral providers. Newly established providers do not report having such cross-sector approaches. These results confirm that sector approaches have long been siloed. It also shows that cross-sector approaches come second, once the basics have been put in place. By emphasising the linkages between sectors and themes, one can expect the SDGs to spur the use of cross-sector approaches over the coming years. 
Figure 3.6. Extent to which providers think that cross-sector approaches are promoted and included in measurement, learning, and reporting systems

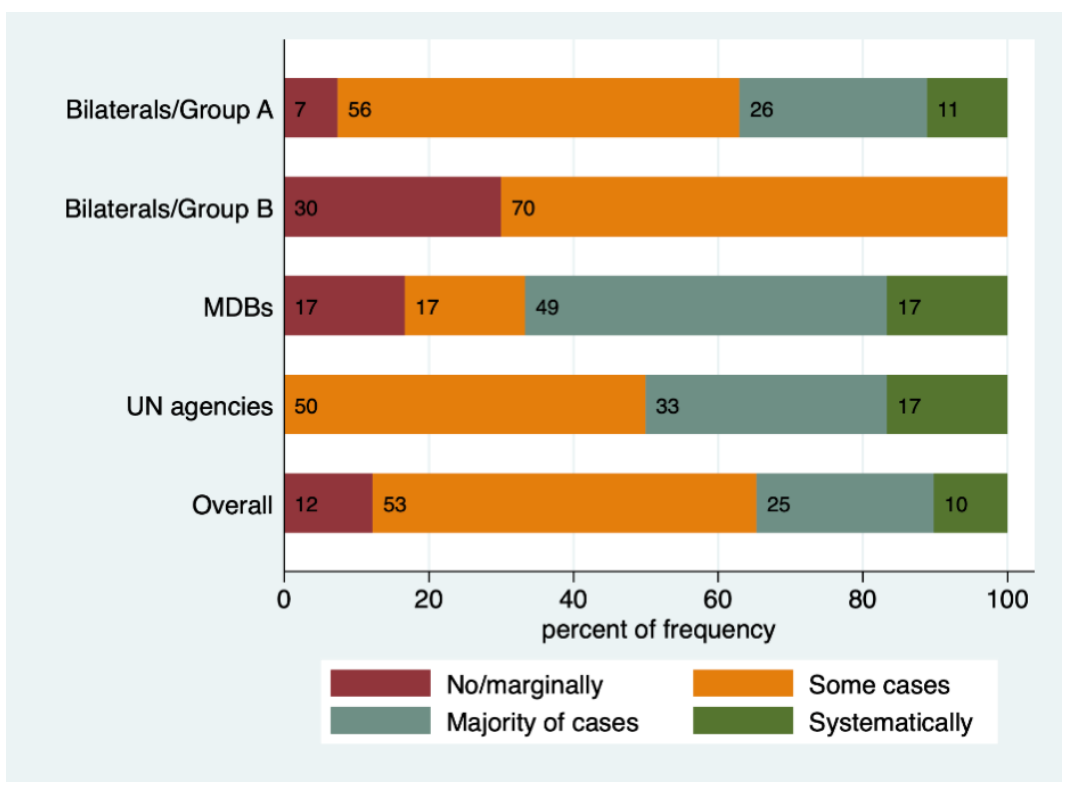

\section{Guiding Principle 2: Adapt to context}

Principle Two of the Guiding Principles on MfSDR - "Adapt to context" - states that "MfSDR systems, methods and approaches should be flexible to allow for them to be tailored to different operational contexts, modalities of engagement, and types of partnerships", (OECD, 2019 $\left.{ }_{[1]}\right)$.

\section{Quality context analysis}

The first key element of Principle 2 invites providers to adapt to each partner country context and local change processes. They also need to be flexible enough to adjust to rapidly evolving situations. This implies relying on quality context analyses that are updated regularly.

According to the survey, most agencies feel that quality context analysis informs results frameworks. On average, such analysis, which allows for a sound understanding of local dynamics and needs, informs the results frameworks systematically $(45 \%)$ or in the majority of cases $(33 \%)$. Most MDBs rely on context analysis systematically $(66 \%)$, while this is the case for only $10 \%$ of recently established bilateral providers (Group B) (Figure 3.7). 
Figure 3.7. Quality context analyses allow for a sound understanding of local dynamics and needs, and inform country- and project-level results frameworks

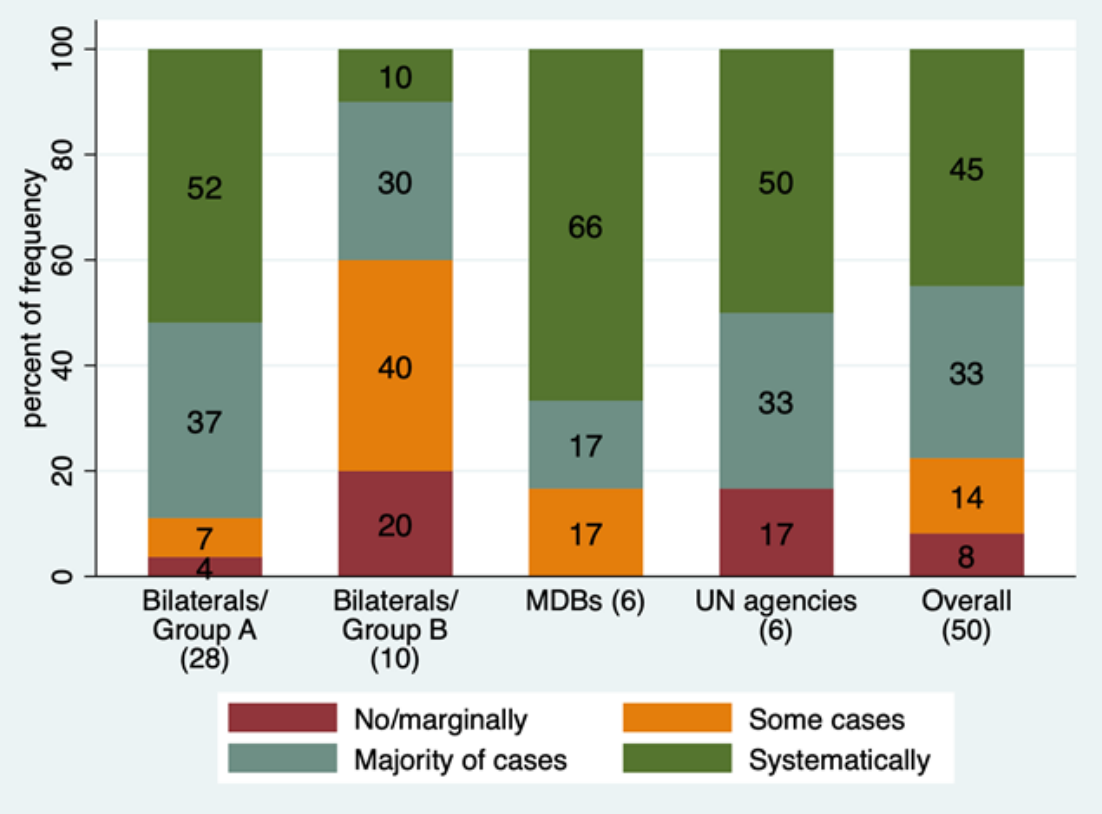

The majority of agencies identify and monitor the conditions for success and risk factors during the implementation phase. One-third of the agencies responded that they did so systematically and a third of them, in the majority of cases. The pattern is logically the same as above, with the MDBs being ahead of the other groups of providers (Figure 3.8).

However, in practice, there are limitations. Some of the additional qualitative responses to the survey note that there is often a difference between policy and practice. For example, one survey respondent notes that 'guidance to staff encourages a contextually driven and adaptive approach to both design and implementation. However, tools, systems and challenges to staff capacity limit the extent to which this happens in practice.' Other providers highlight the scale at which adapting to context is often limited; for example, adapting to context is common during the analysis phase, but less so during the implementation phase; or that an individual project may adapt to changing contexts and needs, but that this is less likely to happen at the country or portfolio levels. 
Figure 3.8. The conditions for success and risk factors in achieving the intended outcomes are identified and monitored during the implementation phase

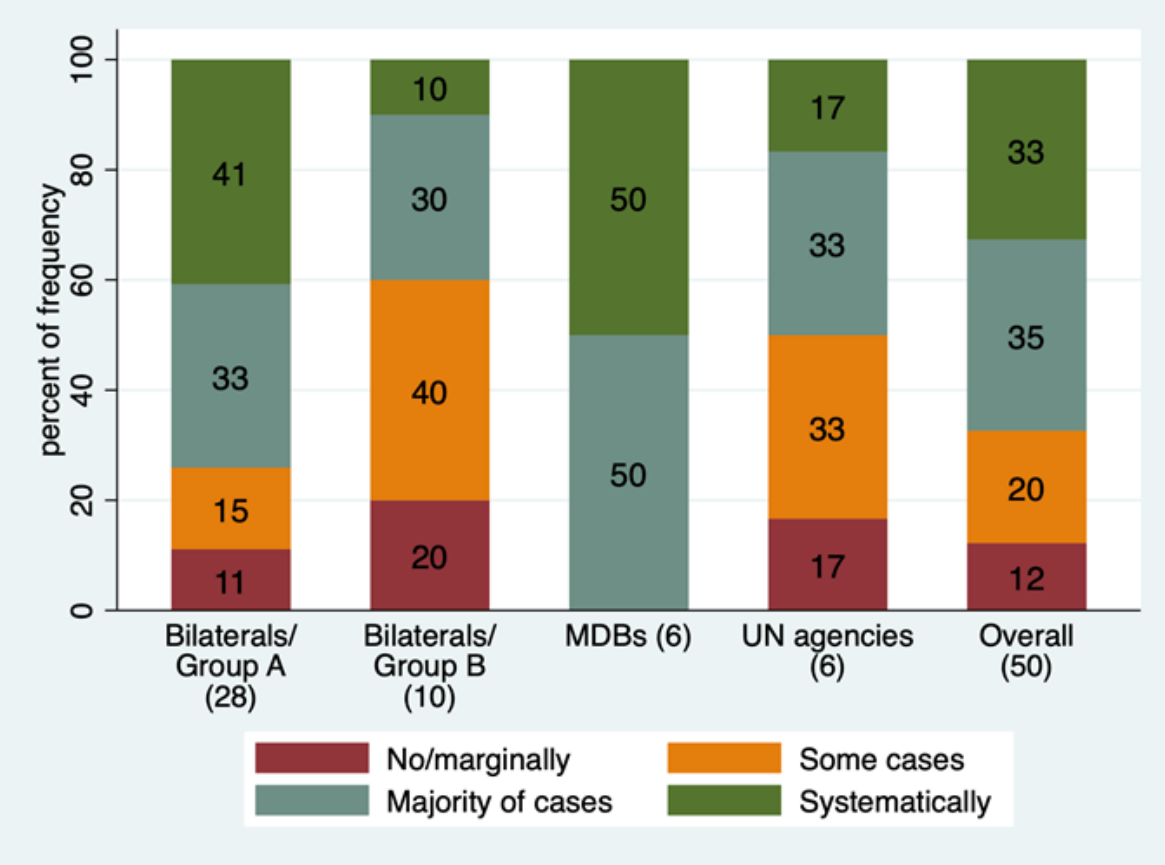

Field-level flexibility and empowerment

Responses to the survey present a relatively positive picture of the extent to which agencies empower staff to adapt to the evidence. Most respondents (78\%) reported that managers have sufficient flexibility to tailor their approaches and processes to each specific context systematically or in the majority of cases (Figure 3.9). The majority of them (59\%) also consider that managers at the country level are focused on long-term outcomes and that they are empowered to pivot a project, in light of changing contexts, so as to achieve this, either systematically or in the majority of cases (Figure 3.10). Recently established providers (Bilaterals/Group B) tend to delegate less authority to managers in the field, which can be explained by the fact that most of them have limited representation in partner countries.

However, having formal flexibility does not mean it will always be used in practice. To illustrate this, the two following comments were made as part of the survey: i) 'managers are empowered to adjust a project's path but may not always take advantage of this empowerment' and ii) 'unfortunately, there is a difference between theory and practice. Long-term outcomes and learning are the focus in theory but during the implementation phase, time and budget pressures lead to less flexibility and adjustment'. 
Figure 3.9. Managers at the country level have sufficient flexibility to tailor their approaches and processes to each specific context (design phase)

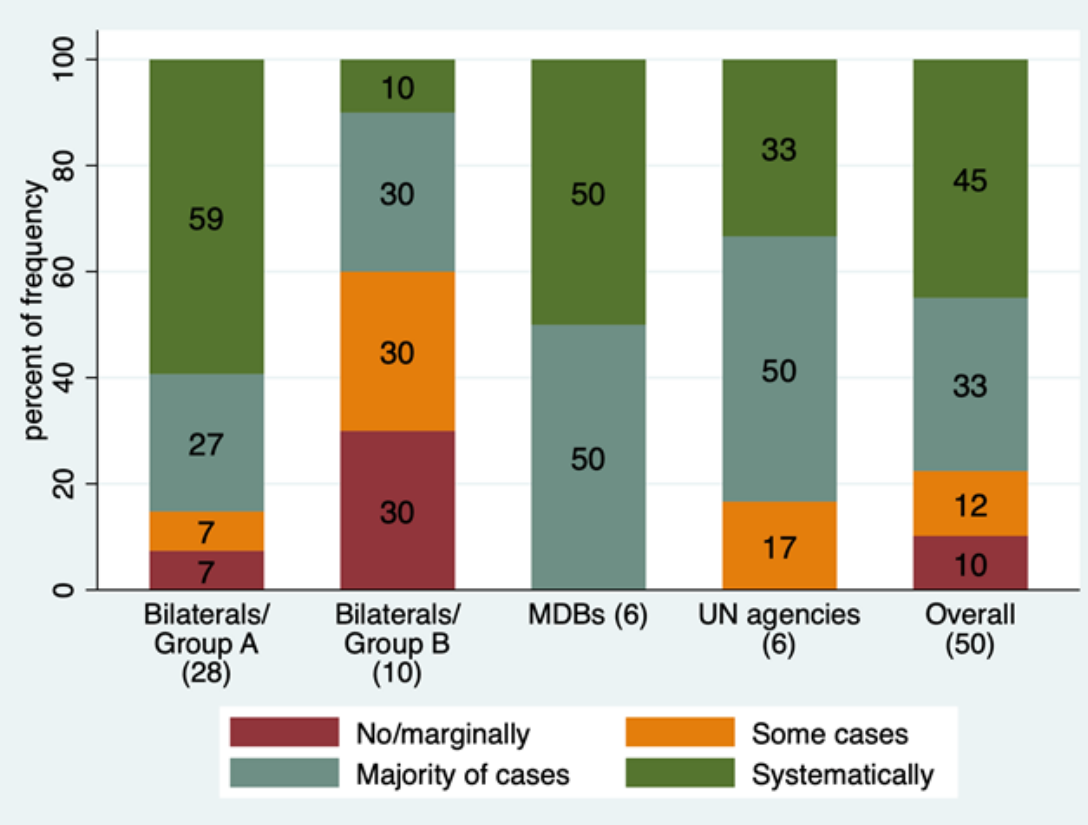

Figure 3.10. Managers at the country level focus on achieving long-term outcomes, they are empowered to adapt a project based on changing local contexts to ensure that the expected outcomes are met (implementation phase)

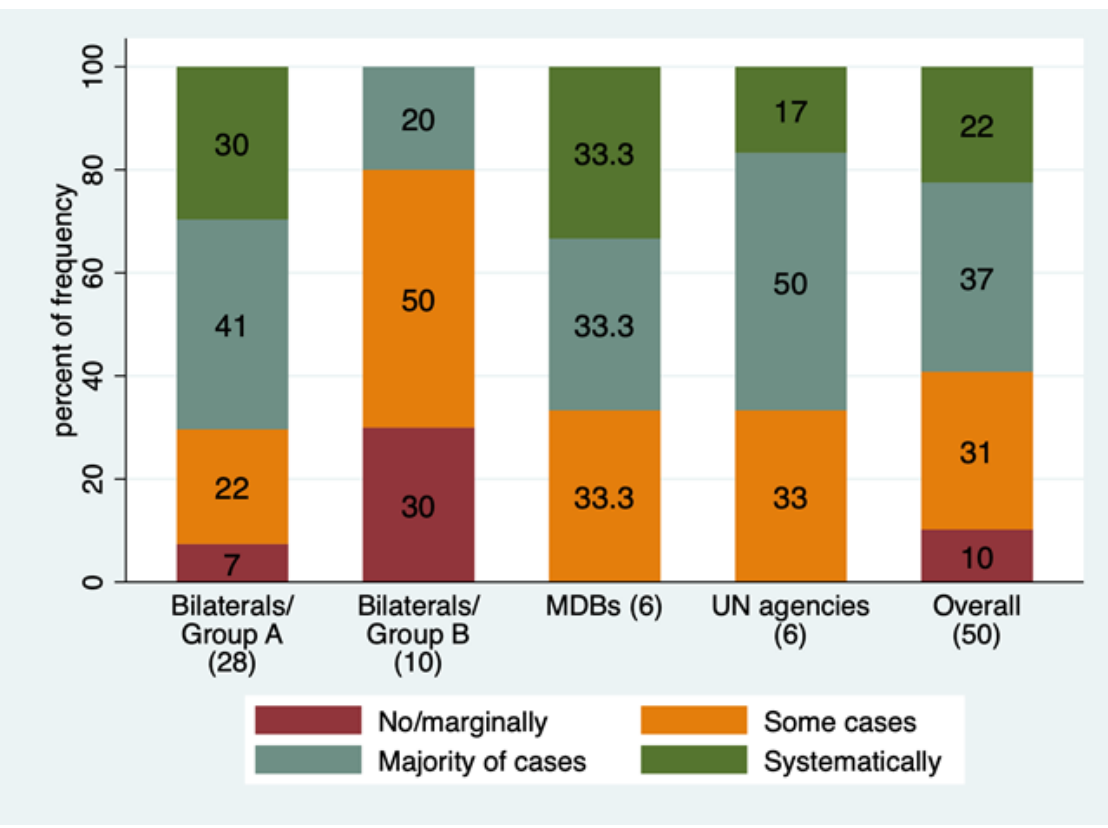




\section{Guiding Principle 3: Enhance country ownership, mutual accountability, and transparency}

Guiding Principle 3 aims to ensure that RBM approaches used by development organisations not only bolster their ability to deliver attributable results, but that these results also co-exist with the development effectiveness principles. As specified in the key elements of this Principle, this requires aligning countrylevel results frameworks with partner countries' results frameworks; synchronising planning cycles at the country level; promoting participatory approaches; maximising the use of and strengthening the monitoring and statistical systems of partner countries; and enhancing mutual accountability and transparency.

\section{Aligning results frameworks}

Alignment with partner country results frameworks is not yet a common practice: More than half of the providers $(55 \%)$ report that they align their country-level results framework with their partner country results framework, including at the indicator level, only in some cases or marginally. The UN agencies are making more efforts to align their country-level results frameworks with their partner country results framework, with half of them doing this systematically and $17 \%$, in the vast majority of cases. Half of the long-established bilateral providers also report that they do so systematically $(7 \%)$ or in the majority of cases (39\%) (Figure 3.11).

This weak alignment can be explained, in part, by the fact that, as seen in the survey responses related to Guiding Principle 1, most providers with a CRF report making efforts to align it to the SDGs at the highest level. All these providers are faced with the tension of reconciling corporate alignment to the SDGs with the need to provide flexibility to field offices so as to align to partner country results frameworks. In a context where not all partner country results frameworks rely on SDG targets and indicators, alignment becomes even more difficult.

\section{Figure 3.11. Country-level results frameworks are aligned with the results frameworks of partner countries, including at the indicator level}

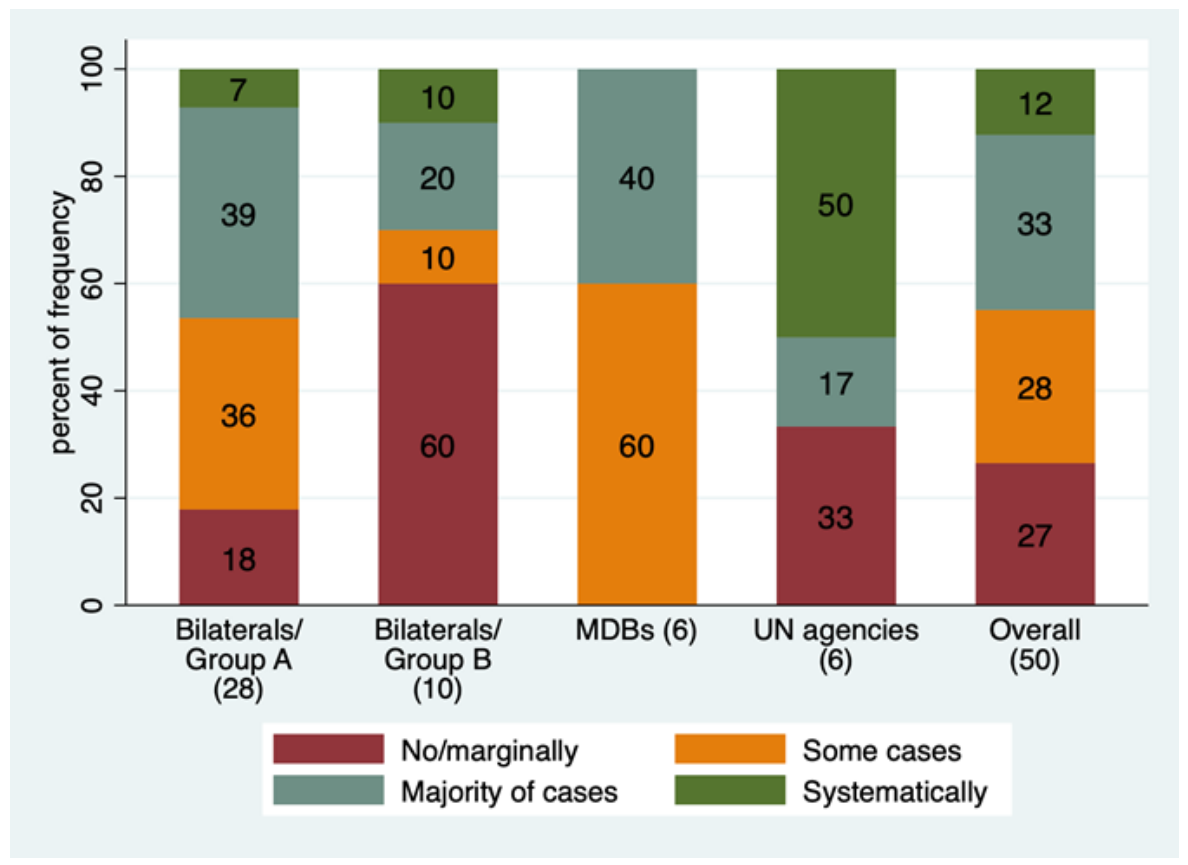




\section{Synchronising planning cycles}

Almost half of providers require or encourage synchronising country level strategies and results planning with the planning cycles of partner countries. This means that country level strategies are updated in accordance with how country priorities and results frameworks have evolved. This synchronisation happens once country strategic frameworks have been updated following an election or once a new national development strategy has been approved. Most development banks $(80 \%)$ and UN agencies $(66 \%)$ tend to do so, while bilateral providers have a more diverse approach (Figure 3.12). The 2019 monitoring round of the Global Partnership on Effective Development Co-operation (GPEDC) confirms that most providers tend to follow the results planning cycle of partner countries, but with an average lag of 2.2 years. This is how much time on average providers need to update their country-level results frameworks once a new national development plan has been approved. In practice, as national plans are updated every four to five years, this means that half the time, many providers who are trying to ensure good alignment are usually catching up with new frameworks (OECD, forthcoming $\left.{ }_{[2]}\right)$. And many others are nevertheless required to follow their own planning cycle at headquarters level.

\section{Figure 3.12. Organisations' planning cycles at the country level are synchronised with the planning cycles of partner countries}

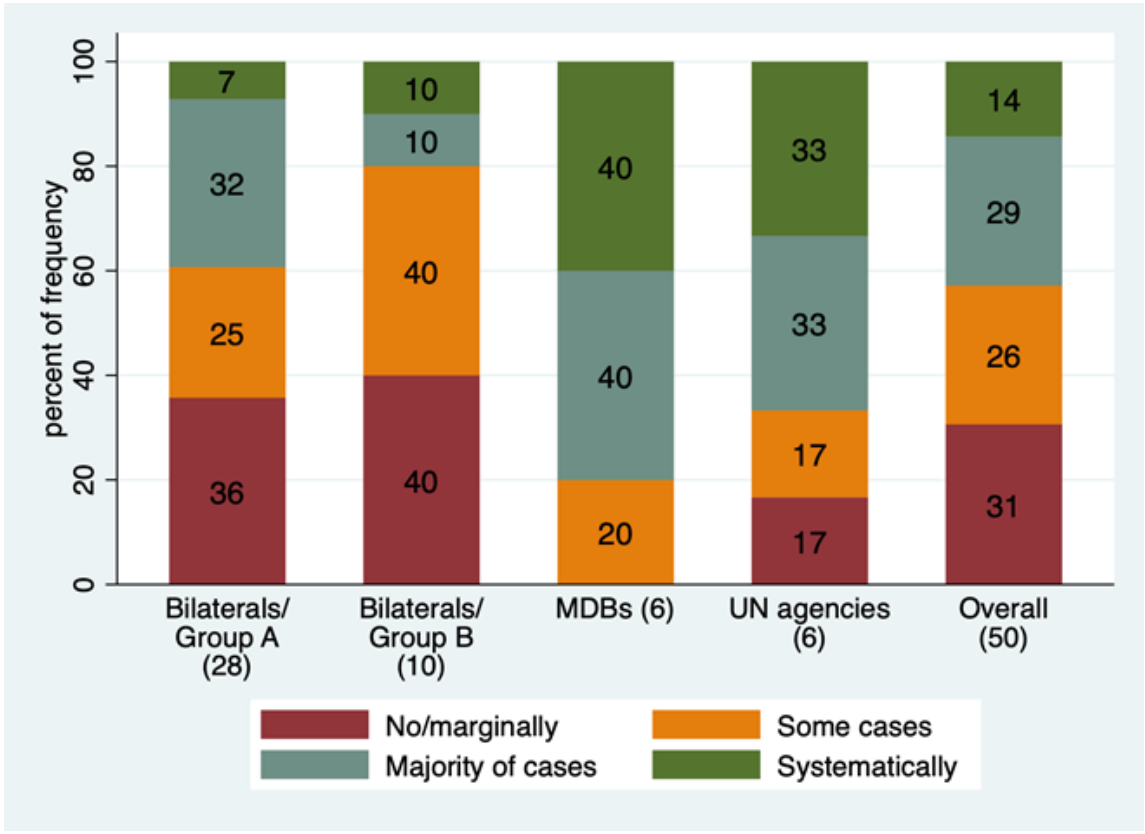

\section{Participatory and inclusive approaches}

Guiding Principle 3 calls on providers to develop country and project level results frameworks in a participatory way and to ensure that problem analysis, design planning, monitoring, evaluation and the use of results is more inclusive.

More than half of the providers report that results frameworks are largely developed in a participatory way, with the involvement of governments in problem analysis, design planning, monitoring, evaluation and in the use of results (56\%). Among two thirds of MDBs and UN agencies, this has already become the norm. Newer bilateral providers (Group B) seek inclusive involvement at a lower rate (40\%) (Figure 3.13). In support of these responses, the 2018 GPEDC monitoring exercise found that broader consultative processes are now more common, with civil society being consulted for $74 \%$ of new strategies. Although the private 
sector and other country actors are consulted only in half the cases, this still represents a positive jump since 2010 (OECD, forthcoming[2]).

Figure 3.13. Country and project-level results frameworks are developed in a participatory way, and involvement is sought in problem analysis, design, planning, monitoring, evaluation and in the use of results

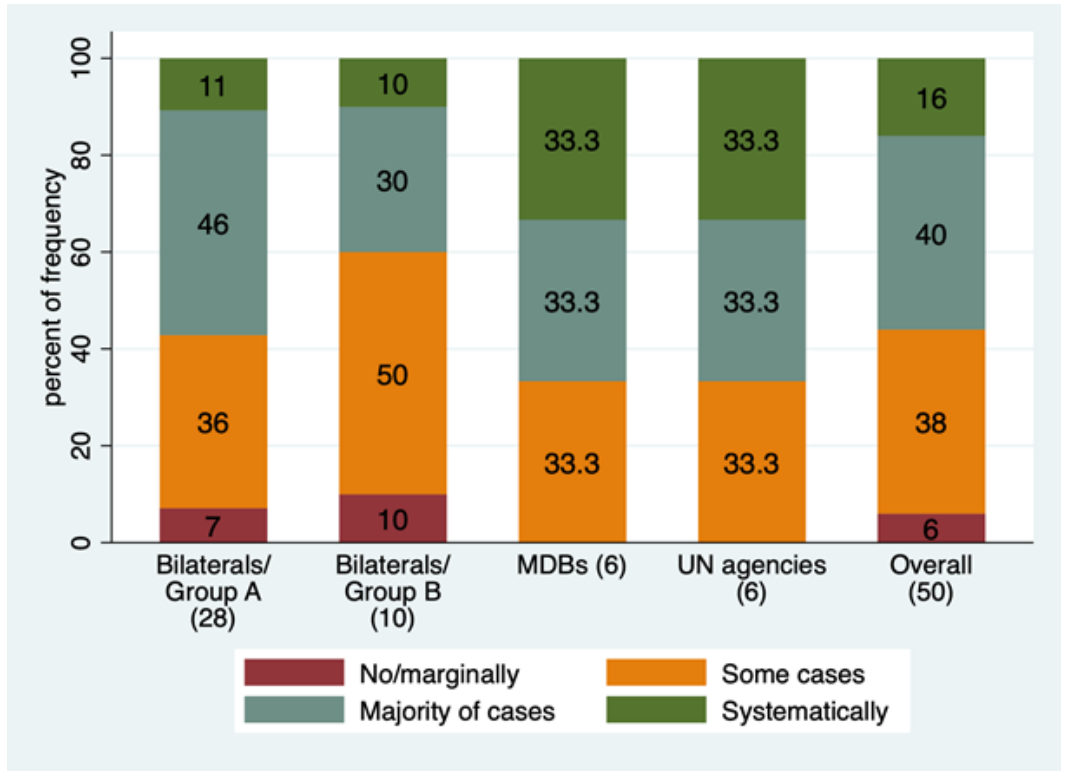

\section{Using and strengthening partner countries monitoring and statistical systems and data}

Around $70 \%$ of providers do not have meaningful monitoring approaches that could make the most of partner countries' monitoring and statistical systems. Only $10 \%$ of providers, but a third of UN agencies that responded to the survey, have systematic monitoring approaches that optimise the use of partner countries' monitoring and statistical systems (Figure 3.14). To explain this, a number of bilateral providers stated that they rely on the monitoring systems of their implementing partners. Others explained that they are still in the early stages of this process of developing country strategies and that so far, monitoring happens only at the project level. A few providers also mentioned that political considerations hamper the use of results information in dialogue with partner governments. Still, most providers emphasise that their goal is to maximise the use of countries monitoring, statistical systems and, to feature results more prominently in dialogue with partner countries. 
Figure 3.14. Monitoring approaches maximise the use of the monitoring and statistical systems of partner countries and their data

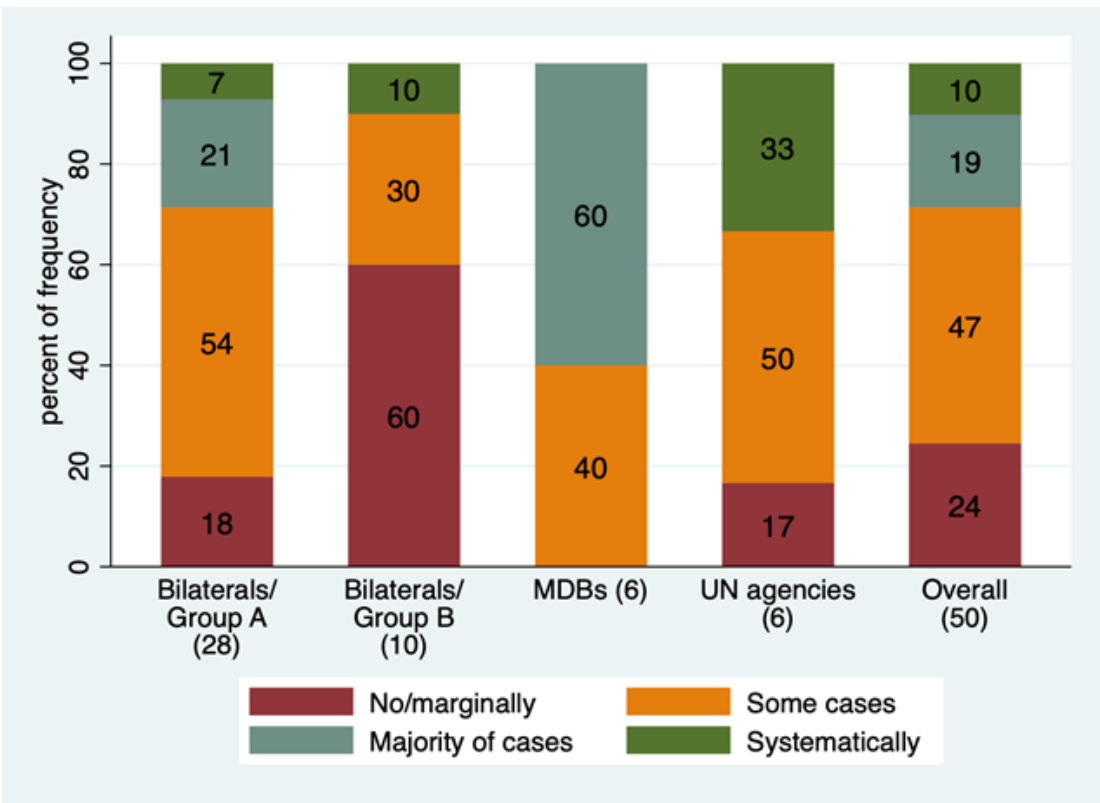

In some cases, the vast majority of providers are strengthening partner countries' statistical and monitoring capacities. This is the case for all MDBs and for $87 \%$ of long-established bilateral providers (Figure 3.15).

Figure 3.15. Efforts are made to strengthen the statistical and monitoring capacities of countries where necessary

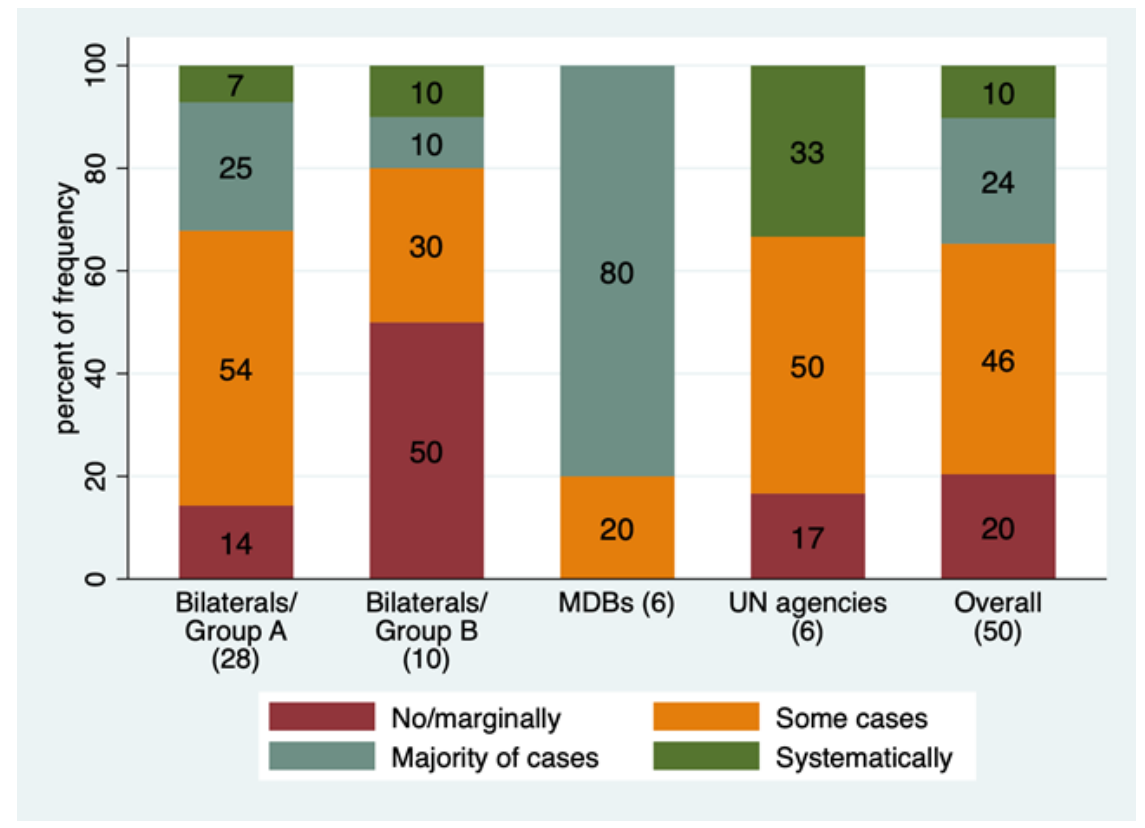




\section{Mutual accountability}

Out of 50 organisations, on average, two-thirds $(62 \%)$ state that mutual accountability is actively pursued fully or in the majority of cases, at a rate of $67 \%$ among MDBs, $68 \%$ among long-established providers and at a lower rate of $40 \%$ among newly established bilateral donors (Figure 3.16). In line with this, the 2018 GPEDC Monitoring Survey, which includes 1179 country offices and embassies, reported that dominant forms of co-ordination and mutual accountability at the country level do at least involve most providers alongside partner governments (33\% of cases), or they include non-state actors such as civil society organisations or the private sector $(23 \%)$. In contrast, $44 \%$ are not fit for mutual accountability since either the providers do not engage $(27 \%)$ or they do so privately (OECD, forthcoming $\left.{ }_{[2]}\right)$.

Figure 3.16. Mutual accountability is actively pursued at the country and project-level with regular dialogue taking place among partners

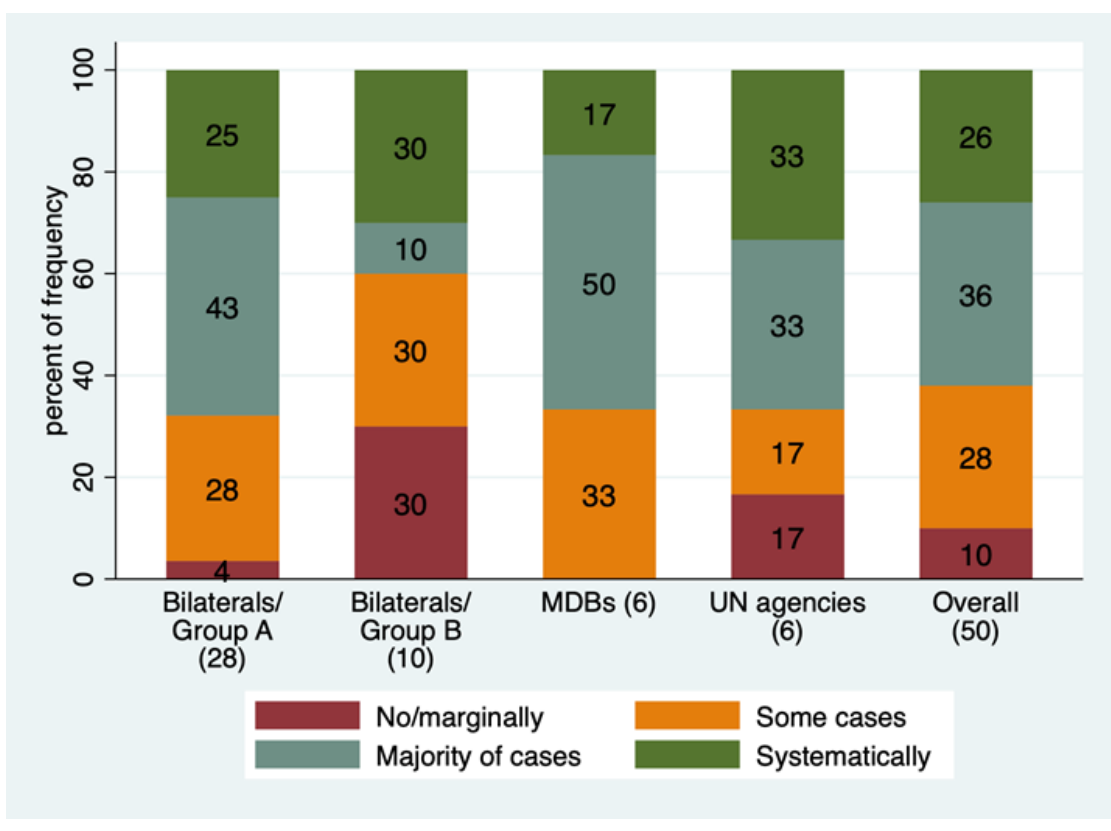

\section{Transparency}

As a pre-requisite for transparency, accessibility to quality and timely information remains limited. Only $12 \%$ of 49 respondents confirm that they systematically publish results frameworks and data with $20 \%$ doing so in the majority of cases. The vast majority of MDBs do publish results frameworks and data, with about half of them doing so systematically. But $14 \%$ of long-established bilateral providers and $30 \%$ of newly established providers do not publish their data (Figure 3.17).

Improving transparency helps make better use of data. A brief comparative analysis shows that whenever results frameworks, information, and data are of a high quality, timely, and made publicly available, then results information from monitoring and evaluation (M\&E) is used for learning and decision making at all levels across the organisation (Guiding Principle 4). 
Figure 3.17. Results frameworks, information and data are high quality, timely, and made publicly available

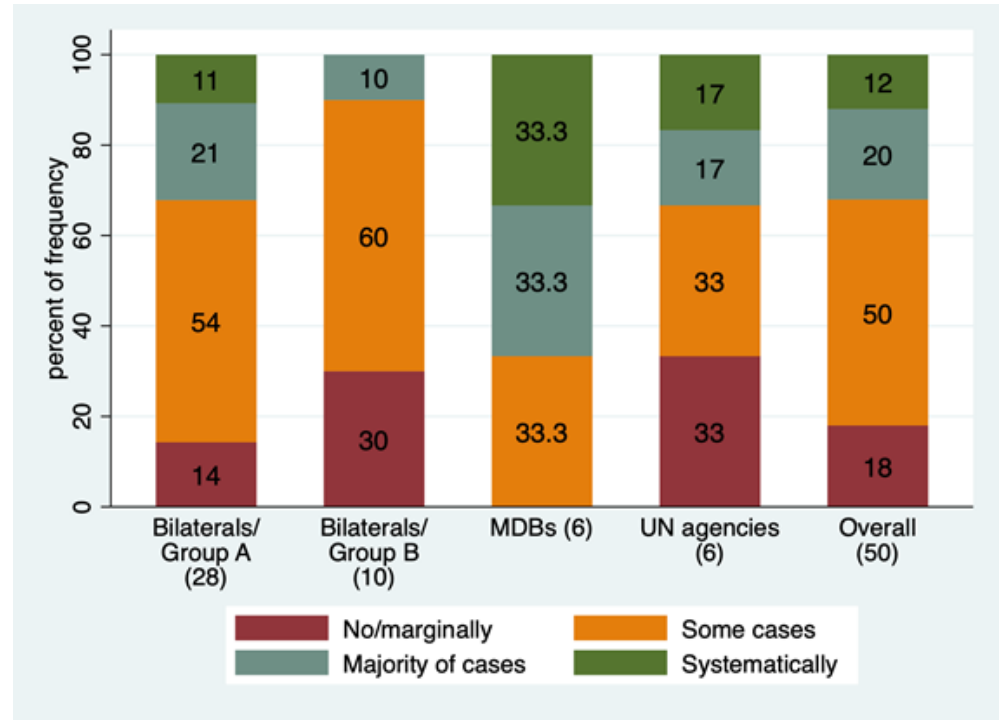

\section{Guiding Principle 4: Maximise the use of results information for learning and decision-making}

Many evaluations of RBM systems point out that what has primarily driven these systems and their reform is accountability. This has had two unintended consequences: a focus on what can be measured easily; and a skewed focus in favour of accountability and reporting, at the expense of learning and decision-making (Vähämäki and Verger, 2019[3]). The MfSDR Guiding Principle 4 aims at balancing the four purposes of RBM: accountability, communication, decision-making and learning.

\section{Using results information for learning and decision making at every level}

For many donors, ensuring that results data is used for learning and decision making is a challenge. The survey shows that only $8 \%$ of providers of development co-operation use results information for learning and decision making at all levels systematically, with $28 \%$ doing so in the majority of cases. This means that nearly two-thirds of providers (64\%) make little or no use of results information for learning and decision making across their institution (Figure 3.18). 
Figure 3.18. Results information from monitoring and evaluation is used for learning and decision making at all levels

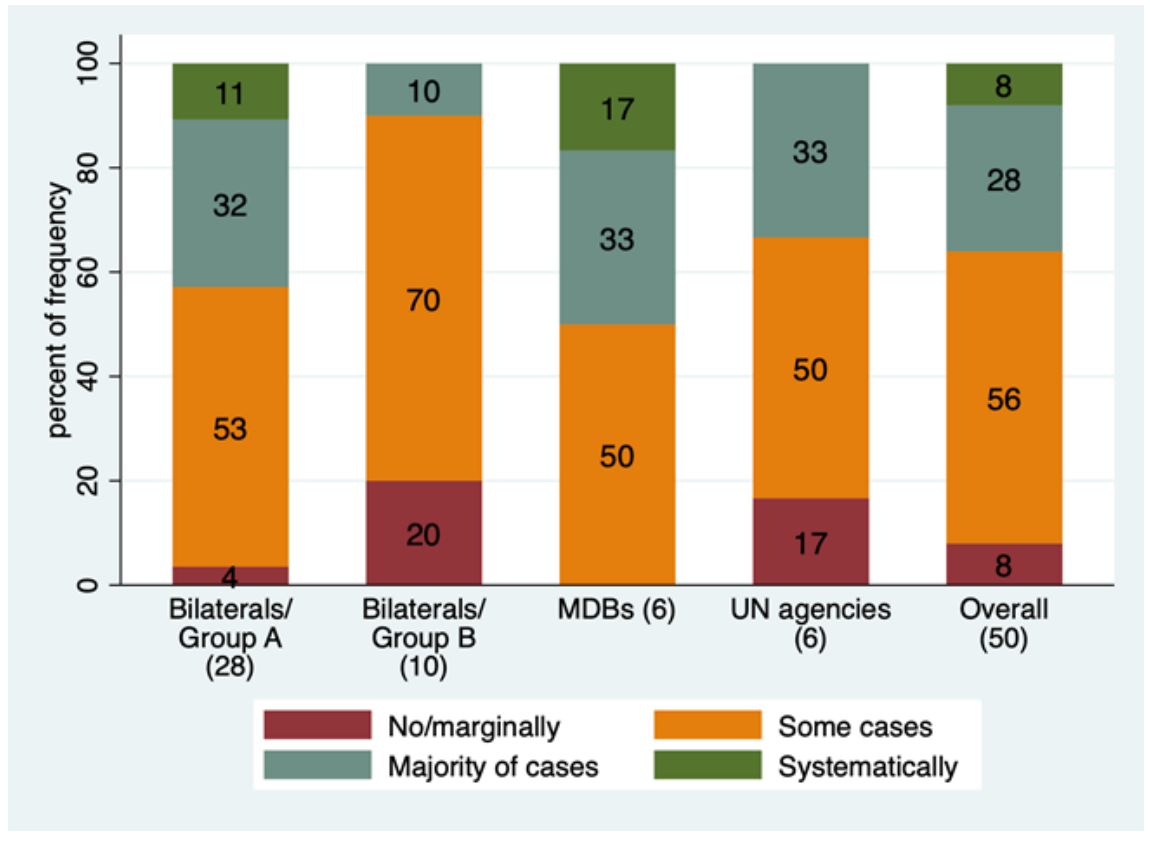

Overall, providers can optimise the use of results information for accountability, communication, steering/decision making and learning more at the country level than at the headquarters level. For the latter, accountability is primarily followed by communication, decision making and to a lesser extent, learning. All types of providers follow this pattern. This comes as no surprise, as accountability has been the main driver for many providers setting up their own results systems. At the country level, providers manage to strike a balance across all four purposes, with equal weight (more or less) being given to accountability, communication and steering/decision making (Figure 3.19). The data suggest newer providers that are still setting up their results systems have yet to aggregate the data they have collected at the project level in order to be able to use this information at the corporate level, either for accountability or for communication purposes.

Learning comes last for all types of providers, both at the headquarters and country levels (Table C.1 and Table C.2 in Annex C). One respondent said that learning is often underestimated and that it requires systematic methods, political will at all levels as well as time and suitable IT and knowledge management systems. The respondent added that learning is also often confused with ex-post evaluation. Although this is disappointing, it is also worth mentioning that learning as a core purpose was emphasised only recently. The informal OECD/DAC survey on DAC members' results approaches that was conducted in 2013 mentioned accountability, communication, decision making and tracking progress as options for using results data. It comes as no surprise that at the headquarters level, accountability was the main purpose for using results data. At the sector and project levels, tracking progress came first. 
Figure 3.19. Use of results information at the headquarters and the country levels

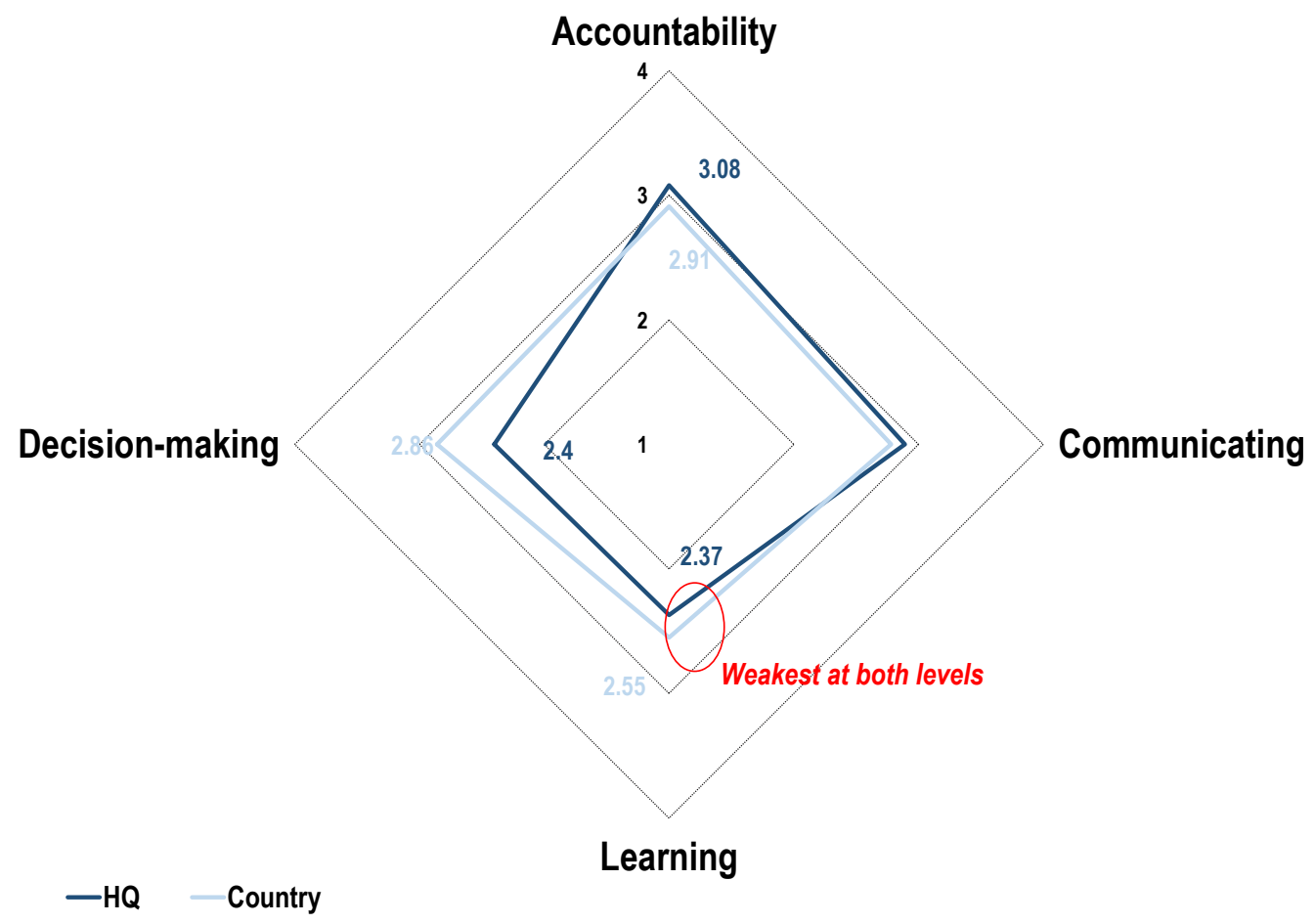

\section{Ensuring that the purpose and use of results information is clearly specified among staff and partners}

The majority of providers ensure that the purpose and use of results data and information are made clear. Out of 50 providers, $58 \%$ responded that this is the case in their organisation either systematically or in the majority of cases. This is more often the case for the long-established bilateral donors and the MDBs ( $72 \%$ and $67 \%$ respectively). Newer bilateral providers are still struggling with building a culture of results within their organisations. Therefore, the purpose and use of results data remains, in the vast majority of cases, fully or partially unclear (80\%) (Figure 3.20). This illustrates that progress is slow. In the 2013 survey, a number of DAC members already felt that it was difficult to say how results information was being used within their organisation. 
Figure 3.20. The purpose and use of results information is clearly defined among staff and partners

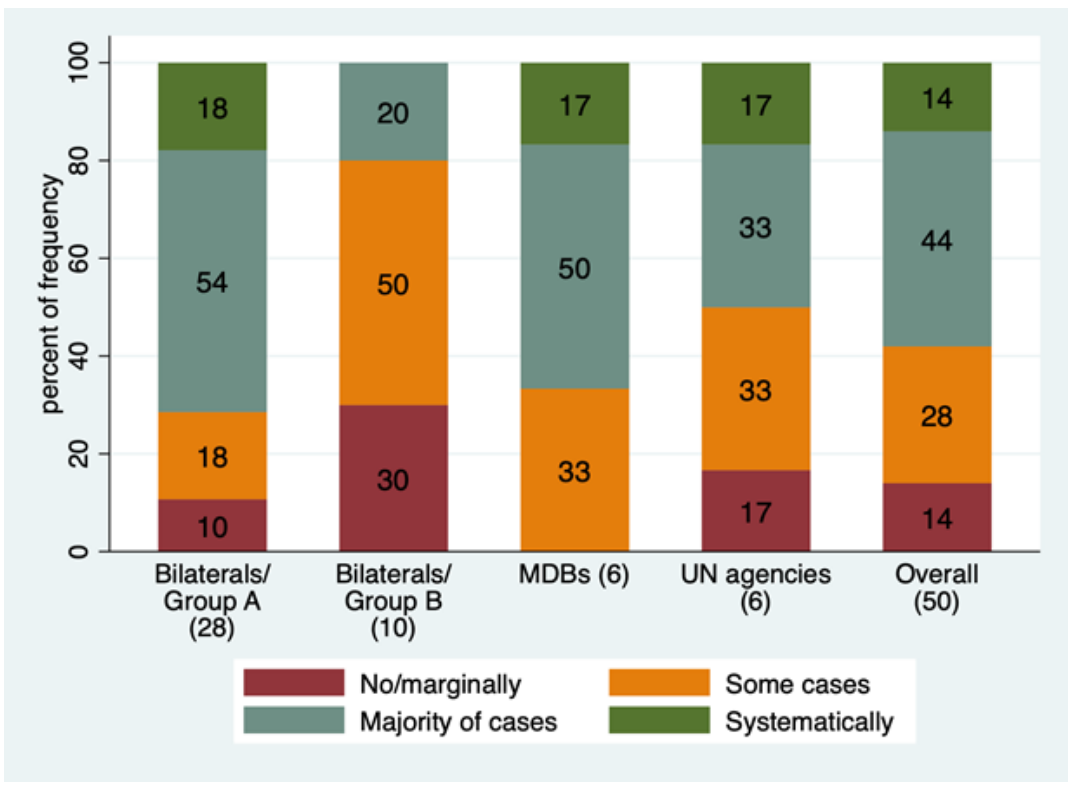

\section{Collection and use of qualitative information}

More clarity is needed on what drives the collection and use of qualitative information. Nearly twothirds of providers (64\%) are using qualitative data to complement quantitative information systematically or in the majority of cases, with multilateral organisations performing better against this indicator than bilateral providers (Figure 3.21). Yet, a number of providers feel that more clarity is required on the use of qualitative information and how such information could be linked to quantitative indicators. Some of the survey's respondents claim that qualitative information is collected only as long as a project uses qualitative indicators. However, although the purpose and use of results information is made clear to staff in internal guidelines, this is not clearly spelled out for each project, which hampers how far qualitative and quantitative information is being used.

Figure 3.21. Qualitative information is collected to complement quantitative data

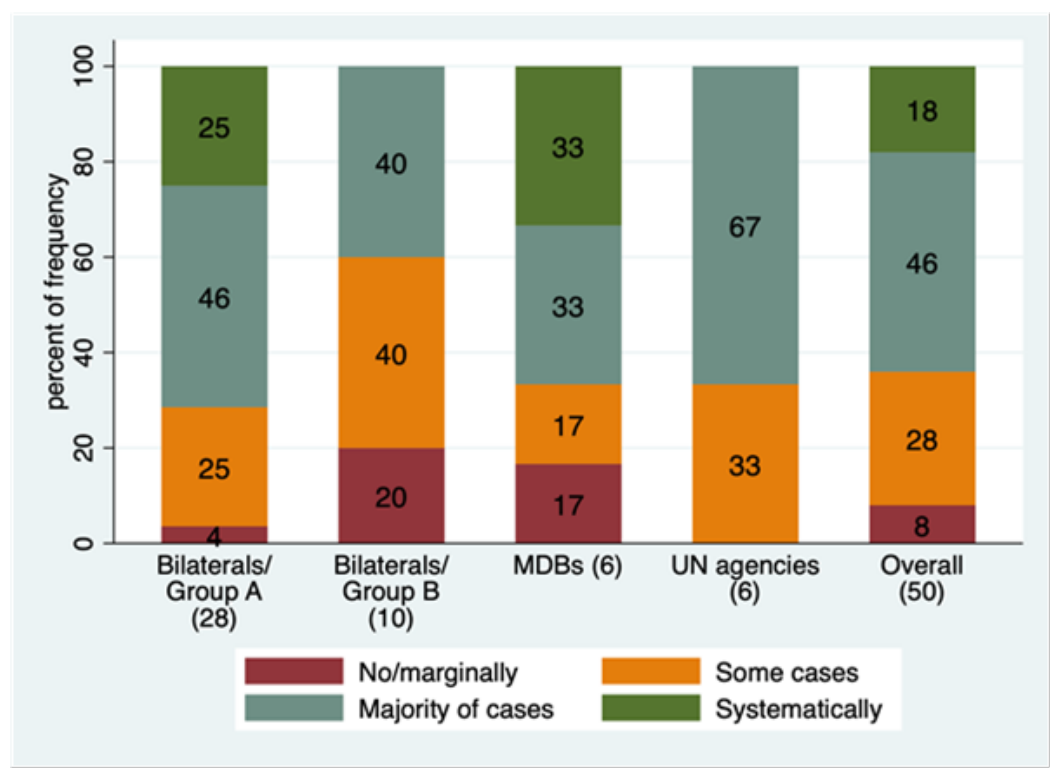




\section{Guiding Principle 5: Foster a culture of results and learning}

This Principle, which stands for the renewal of the results agenda, invites organisations to promote and sustain a culture of results through consistent leadership, appropriate guidance, tools and capacity building as well as by using proper incentives.

\section{The role of leadership}

For the majority of providers, leadership does not fulfil its role in fostering a culture of results. On average, only four out of ten providers feel that leadership communicates the purpose of RBM and that it uses results information to influence the overall programme management and policy landscape either systematically or in the majority of cases. This is more common among UN agencies $(66 \%)$ and MDBs $(50 \%)$ than for long-established (39\%) or newly-established bilateral providers (20\%) (Figure 3.22). The feedback from respondents suggests that while leadership across both older and newer bilateral donors acknowledge RBM's crucial role, elevating the results approach to the corporate and the programme levels remains a challenge, and that its dissemination also needs to be enhanced among country offices and implementing partners.

Figure 3.22. Leadership communicates the purpose of results-based management and uses results information for overall programme management and to influence policy at the political level

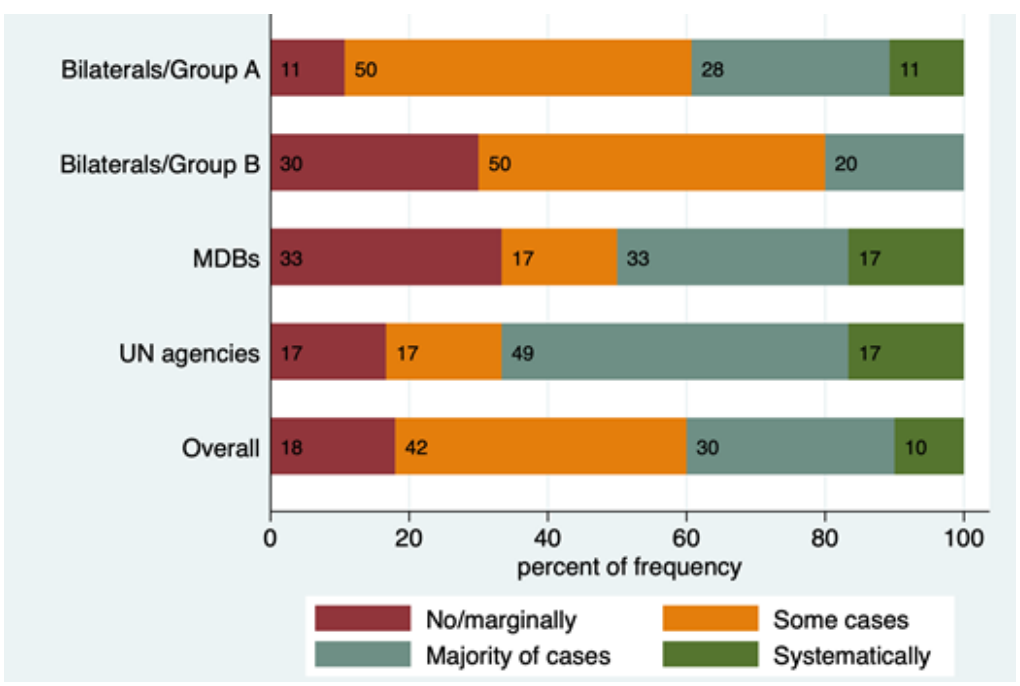

Most providers feel that appropriate guidance and tools are lacking. Only one-third of organisations responded that leadership has put in place appropriate guidance, incentives and resources for MfSDR systematically or in the majority of cases. According to the survey, the organisational approach to results seems to be more developed among MDBs ( $50 \%$ have an organisational approach to results). Two-thirds of UN agencies and long-established bilateral providers are still struggling to put the right system in place. This is even more the case for newly established bilateral providers (80\%) (Figure 3.23).

Comments made by bilateral providers suggest that building staff awareness and capacity are a steppingstone for strengthening a provider's results focus in development co-operation. Guidance, incentives and resources come next, as well as the need to work harmoniously. This is not so frequent for long-established bilateral providers ( $40 \%$ responded that this happens systematically or in the majority of cases). It is an exception among newly established providers (10\%). Many members are struggling with finding the right incentives. One respondent mentioned that often, leadership express interest in RBM and focus on guidance, discussions and dialogue, but specific incentives for staff are lagging behind. Another respondent mentioned the downside to increasing the aid budget has been that establishing a culture of results has been slowed 
down, given the number of new staff that have had to be absorbed and incentives reoriented towards achieving financial targets.

Figure 3.23. Leadership promotes a culture of results and learning by providing appropriate guidance, tailored incentives and resources for managing for sustainable development results

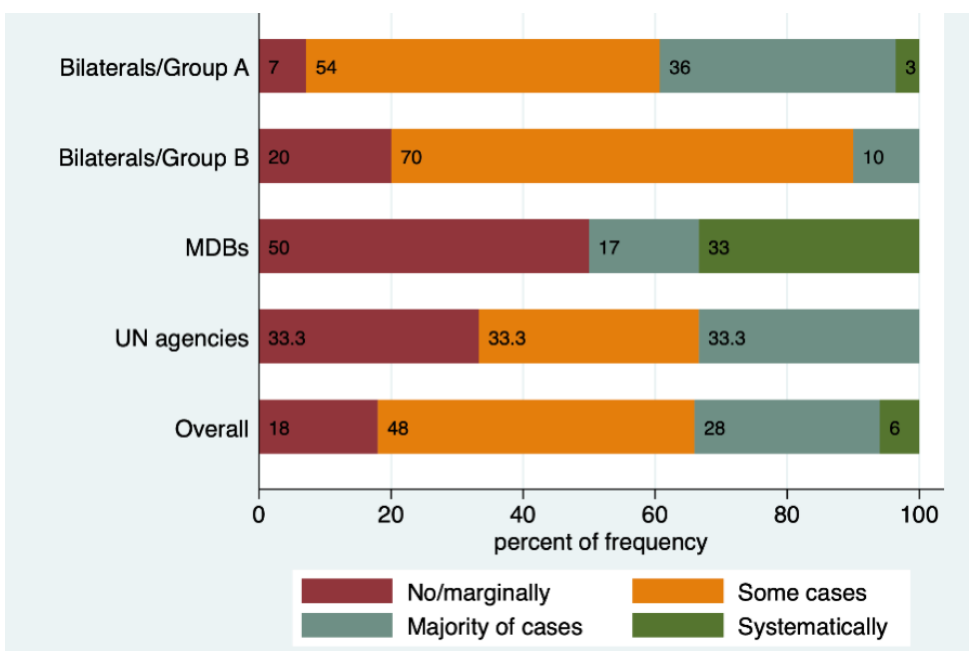

\section{Training opportunities}

Training in RBM approaches tends to increase among more-established organisations. While newly established providers offer very limited training opportunities ( $45 \%$ are not providing training and $55 \%$ only in some cases), half of the other providers offer training systematically or in the majority of cases (Figure 3.24). Newly established providers attributed this to staffing constraints in smaller organisations. Although there are learning platforms as well as opportunities for further education, staff are often overworked, serving multiple roles so that RBM can compete with other fields of expertise. On the other hand, large numbers of consultants working on projects within UN agencies make it challenging to train all staff properly and in a systematic manner. 
Figure 3.24. All relevant staff are trained in results-based management approaches and methods

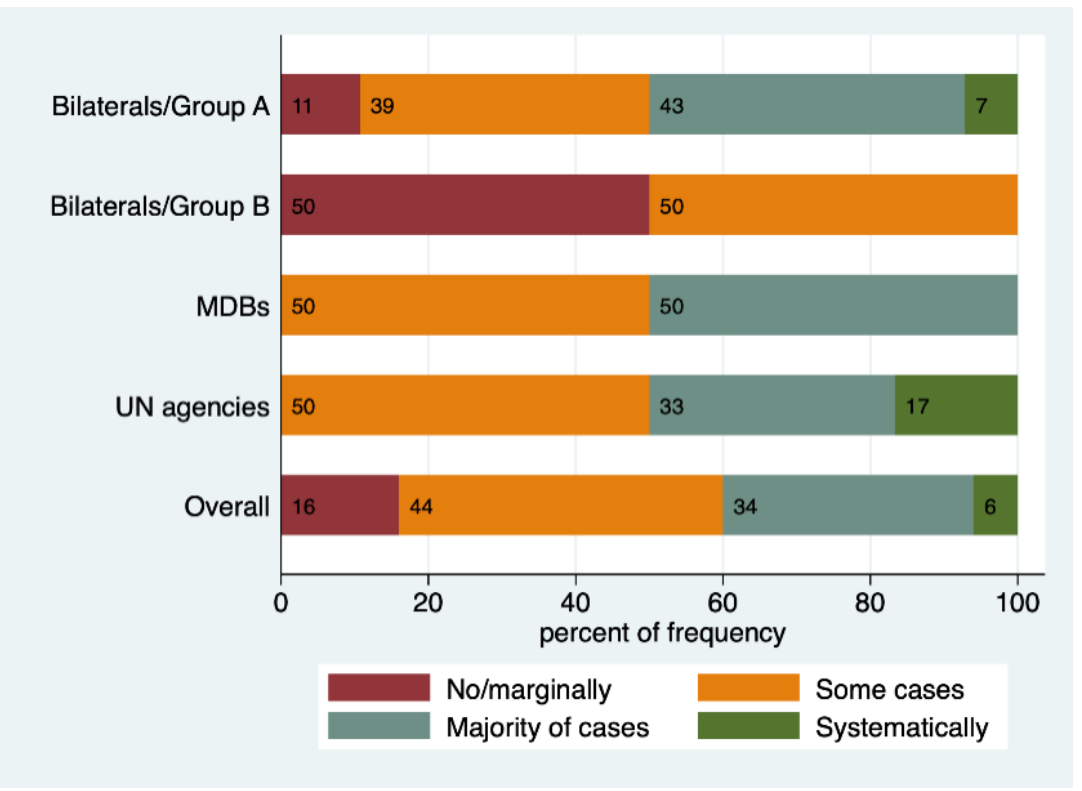

\section{Scope for peer learning}

Half of the providers offer opportunities for peer learning on successes and failures. Such opportunities seem to be less frequent among UN agencies, with two-thirds of respondents noting that there is no or marginal room for peer learning on successes and failures or, that this happens only in some cases. Comments from bilateral agencies suggest that even when there is a strong commitment from leadership to the RBM approach and its communication, it takes time to implement reform systematically, so as to create the time and space for providers to learn from the results information (Figure 3.25).

Figure 3.25. There are mechanisms for peer learning on successes and failures

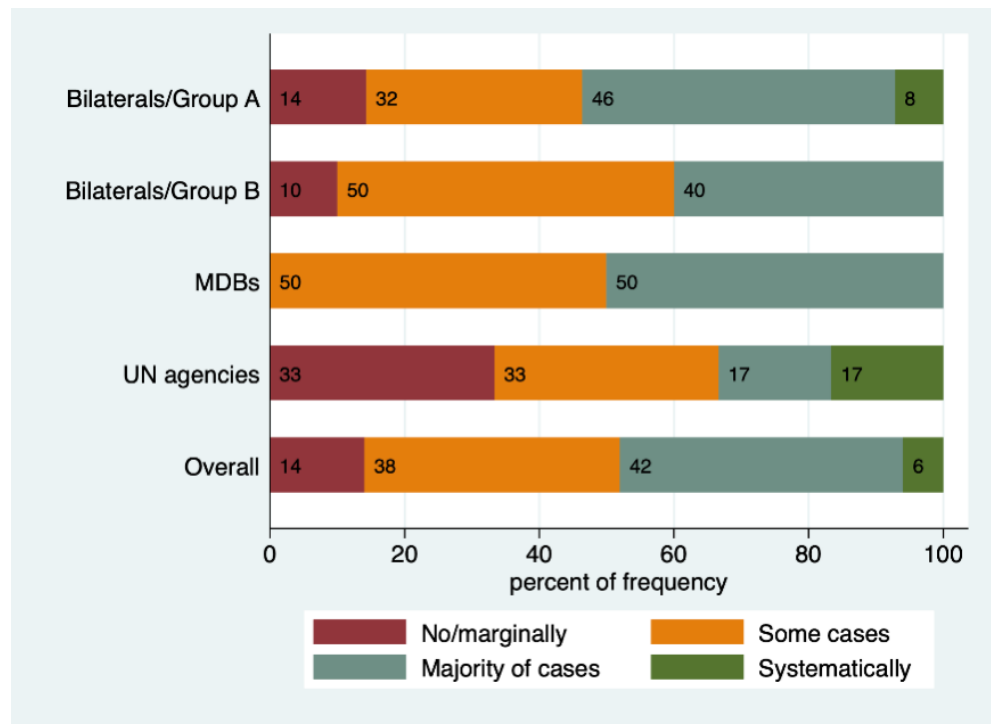




\section{Experimentation in support of innovation}

Experimenting to promote innovation is not yet common practice. Nearly two-thirds $(63 \%)$ of providers do not encourage experimentation or they do so only in some cases. The UN agencies stand out, with $80 \%$ of them valuing careful experimentation in favour of innovation either systematically or in the majority of cases. Bilateral providers said that while their organisation certainly values careful experimentation in support of innovation, putting these approaches into practice has proven to be far more complicated. It requires skills and resources (Figure 3.26).

Figure 3.26. The organisation values careful experimentation in support of innovation as a way to foster better results

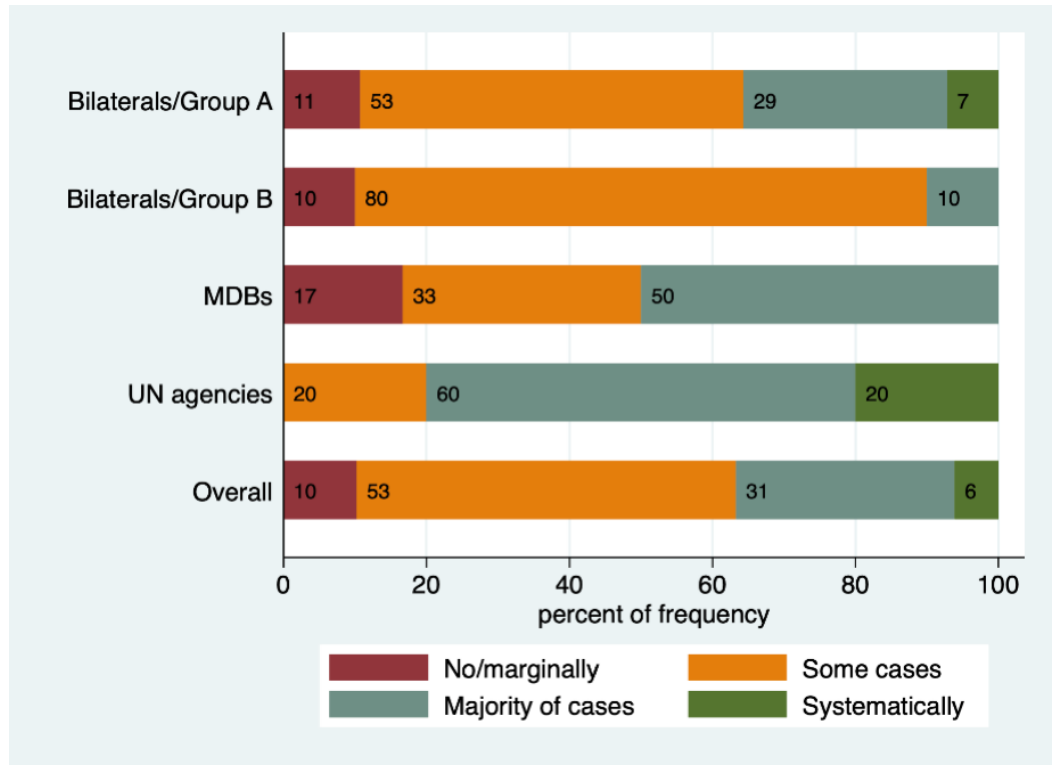

\section{Guiding Principle 6: Develop a results system that is manageable and reliable}

Some of the challenges identified by members with regard to Guiding Principle 6 include: establishing IT systems that can collect, process, and share results information; measuring results accordingly for accountability, reporting, decision making, and learning needs across levels; and providing adequate guidance for staff and partners using results systems and data.

\section{Monitoring and evaluation (M\&E) systems}

For the majority of providers $(60 \%)$, M\&E systems are not adequately resourced. MDBs and UN agencies claim to have M\&E systems that meet the needs of their organisation in some or most cases, although this does not happen systematically. Interestingly enough, newly established providers (Group B) score significantly higher in this category with $50 \%$ stating that they have adequate M\&E resources systematically or in the majority of cases as opposed to long-established providers in Group A (36\%) (Figure 3.27). This difference in perception could be explained by the fact that recently newly established providers have dedicated effort and resources to setting up their M\&E systems, since having an evaluation function is one requirement for becoming a member of the DAC. Meanwhile, more-established providers tend to see the limits of the data they can get through their M\&E systems as they increasingly try to make more use of results information for steering and learning. 
Figure 3.27. Monitoring and evaluation systems are adequately resourced to meet the needs of organisations

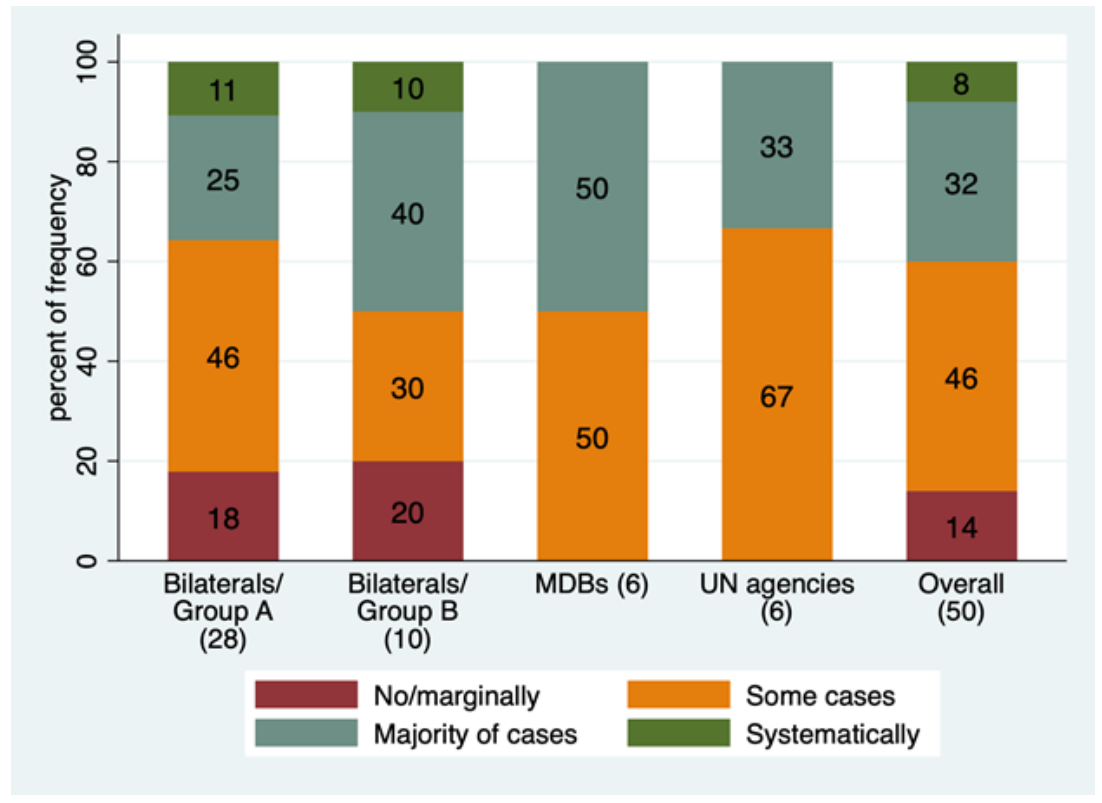

\section{Quality of evidence}

The majority of providers think their M\&E systems produce credible quantitative and qualitative evidence, which adequately meets their needs. Multilateral organisations responded less optimistically, saying that this never happens systematically, but only in most cases $(50 \%)$ or in some cases $(50 \%)$. Meanwhile, $65 \%$ of long-established providers (Group A) said this was true in the majority of cases unless it was done systematically. Newly-established providers (Group B) lag behind with only $40 \%$ saying that M\&E systems have been set up properly (Figure 3.28 ).

Figure 3.28. Monitoring and evaluation systems are set up to produce credible quantitative, qualitative data as well as evidence that meets the needs, capacities of development organisations, their partners

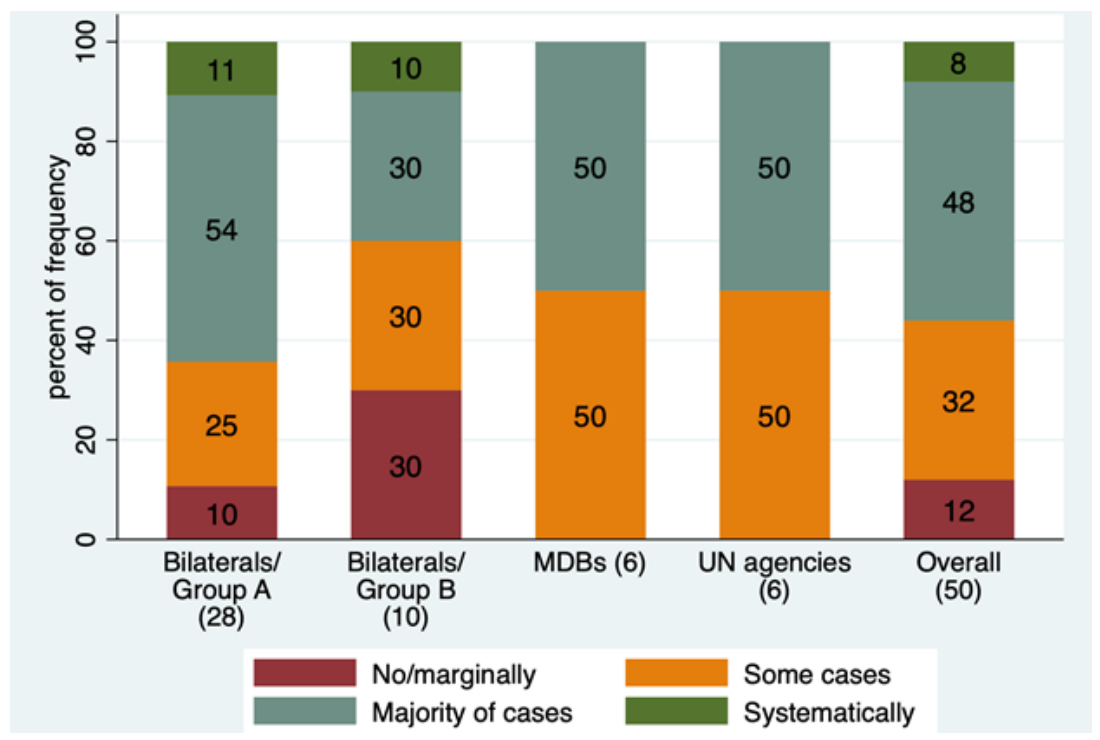




\section{Information systems}

Less than half of providers (38\%) feel as though their RBM practice is supported by adequate information systems. Therefore, for all providers, the biggest impediment for developing a results system that is manageable and reliable is the lack of adequate IT systems that are able to manage quantitative and qualitative results information. Across all categories, only long-established providers (Group A) state that in $7 \%$ of cases, these systems are established systematically. However, MDBs and UN agencies fare slightly better (with $67 \%$ and $50 \%$, respectively, having adequate IT systems in the majority of cases) than bilateral providers (who account for $39 \%$ and $10 \%$ for Groups A and B, respectively). (Figure 3.29). A shortage of staff is also mentioned as one hindrance: even though some providers were able to put a new IT system in place, this has proven to be far too complex for staff who were already stretched in the first place. In any case, high tech digital tools can be difficult to implement, and it takes significant amount of time as well as resources to set them up properly.

\section{Figure 3.29. Results-based management is supported by adequate information systems, and it has the ability to manage quantitative and qualitative results information}

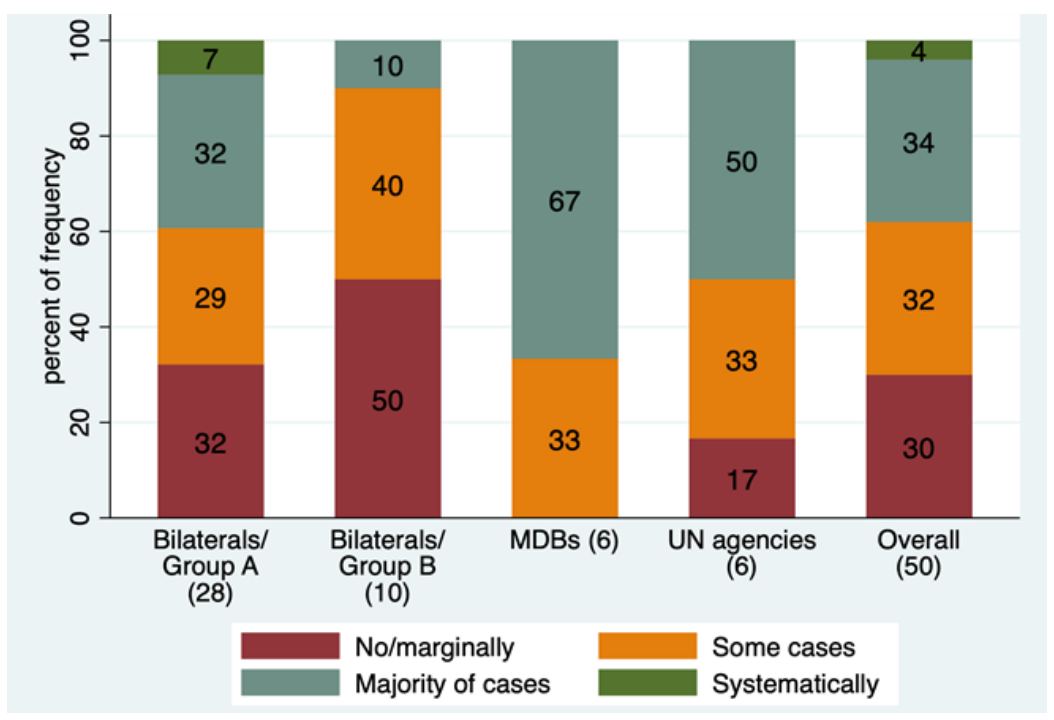

\section{Results-based management systems}

Most providers feel that the overall RBM system is not user friendly, and that it is not reviewed regularly enough to meet its purpose (64\%). Even among UN agencies, which score significantly better in other components of Guiding Principle 6, RBM systems lag behind (66\% state that the overall RBM system is not user friendly at all or, only in some cases). This is even more pronounced for newly established bilateral providers (Group B) where in $80 \%$ of cases, RBM systems are not perceived as user friendly and/or are reviewed regularly (Figure 3.30). Some respondents said that even though they are continuously developing their RBM systems to make them more manageable and fit-for-purpose, this is not done in a systematic manner, which causes delays. Other respondents said that as a whole, digitisation remains a challenge to making M\&E systems more user friendly and reporting more straight-forward. 


\section{6}

Figure 3.30. The overall results-based management system is user friendly, lean and reviewed regularly to fit its purpose

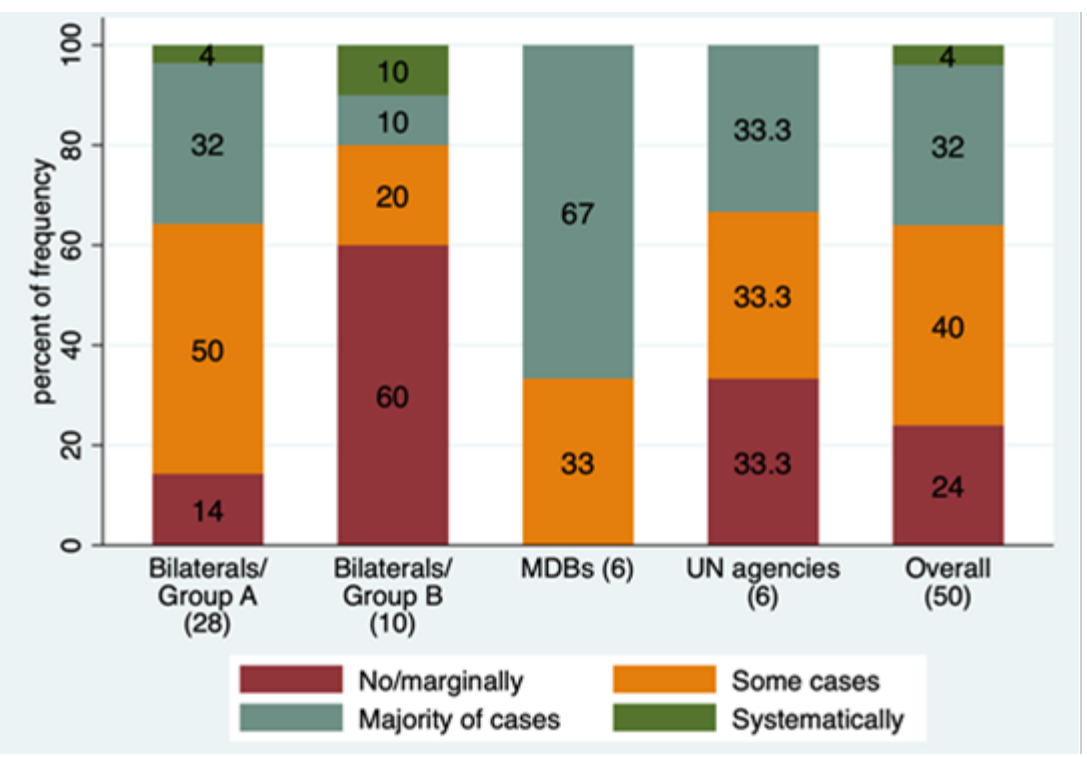




\section{Correlations between Guiding Principles}

A number of key elements within the Guiding Principles are interdependent, which is revealed in an analysis of responses to the survey. This section explores correlations ${ }^{4}$ that are moderate or significant. These show positive synergies that organisations could use to reinforce their approach to RBM. The first three Guiding Principles define the vision driving an RBM system and data from the survey reveals that there is no significant or moderate correlation between them. This chapter considers the relationships between each of the first three "visionary" Guiding Principles with Guiding Principles 4, 5 and 6, which focus on what is required to generate and use results information effectively, ending with correlations between these three 'enabling' Guiding Principles.

\section{Correlations between Guiding Principle 1 and key elements of Principles 4, 5 and 6}

Guiding Principle 1 on 'Supporting sustainable development goals and desired change' sets the overall vision for RBM, and all the other principles more or less stem from this vision. Correlations are particularly strong with Guiding Principle 4 on maximising the use of results information for learning and decision making and Guiding Principle 6 on 'Developing a results system that is manageable and reliable'. Responses to the survey do not show strong correlations with the key elements of Guiding Principle 5 (Foster a culture of results and learning).

The use of established methodologies is correlated with the quality of the RBM system. As shown in Figure 2.4, the use of established methodologies such as theories of change and logical frameworks to define the results chain at the corporate level depends on the extent to which the overall RBM system is lean, user-friendly, and reviewed regularly (key element of Guiding Principle 6). Obviously, a management decision of developing dedicated methodologies at the core level translates into establishing a system that is able to generate all the reliable information required for the results-frameworks to be used effectively.

Having results frameworks translate into more frequent use of results information. A strong correlation exists between the prevalence of results-frameworks at the sector level, and the use of results information for learning and decision making (Guiding Principle 4). Creating a results-framework creates demand for data, as well as generating data that can be used for different purposes.

Cross-sector approaches are correlated with the use of results information. There is also a strong relation between the extent to which cross-sector approaches and their inclusion in measurement, learning and reporting systems are promoted and, whether or not results information is used for learning and decisionmaking at all levels. Meanwhile, the prevalence of cross-sector approaches is also correlated with the extent to which the purpose and use of results information are clearly explained among staff and partners. This suggests that organisations with the ability to address complex cross-sector approaches need more specific results information and that they are likely to make more use of this information. 
Figure 4.1. Correlations between Guiding Principle 1 and key elements of Guiding Principles 4 and 6

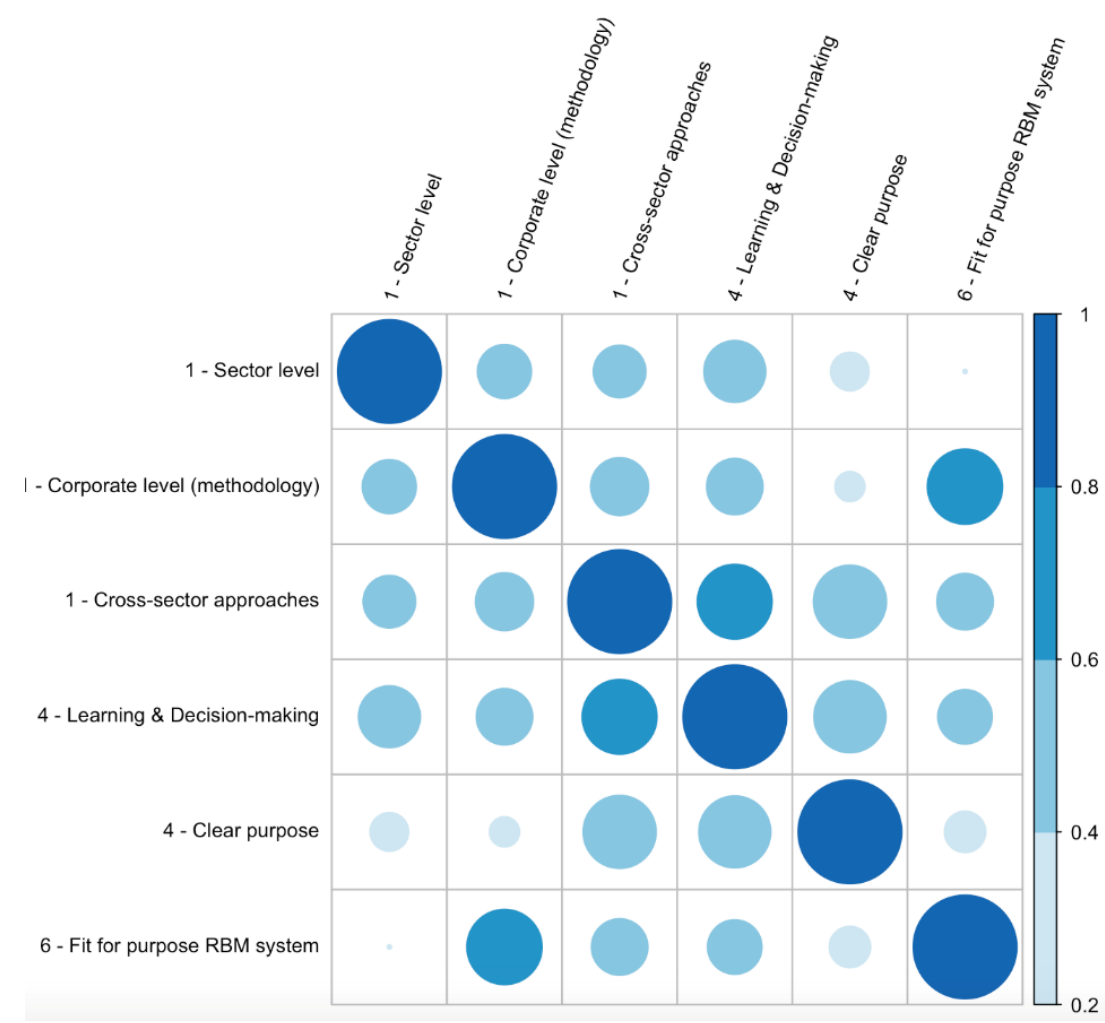

Note: Correlation coefficient used is Spearman's Rho, where values of 0.6 and above indicate a strong relationship as well as a darker colour and larger circles.

\section{Correlations between Guiding Principle 2 and key elements of Principles 4, 5 and 6}

Guiding Principle 2 on adapting to context is strongly correlated with Guiding Principle 4 on using results information. How far adapting to change is facilitated depends on whether results information is used for learning and decision making at all levels. More specifically, there is a strong correlation between:

- whether managers at the country level focus on achieving long-term outcomes and are empowered to adjust a project based on changing local contexts so as to ensure that they meet their outcomes.

- how far, at the country level, results information is used for i) learning and ii) steering and decision making. (Figure 4.2).

A correlation, even if it is weaker, also exists between results information being used for learning and decision making at all levels and managers at the country level having enough flexibility to tailor their approach and their processes to each context, specifically during the design phase. Such moderate correlation also exists between use of results information and how far managers can focus on achieving long-term outcomes and how far they are empowered to adjust a project based on changing local contexts, so as to ensure that they meet their outcomes during the implementation phase.

There is a correlation between the use of quality context analysis and appropriate training on RBM. Some key elements of Guiding Principle 5 are very relevant for creating an appropriate culture, one that supports adapting to a specific context. Responses to the survey specifically reveal how far quality context analysis informs country and project level results frameworks. This depends on whether all relevant staff are trained in approaches to RBM methods. 
Surprisingly, the survey does not reveal a strong correlation with Guiding Principle 6. One could expect that when M\&E systems are set up to produce credible quantitative and qualitative data and evidence that meets the needs and capacities of the development organisation and its partners (Guiding Principle 6), providers are more likely to adapt to different contexts with more flexibility, by adjusting results framework to local dynamics. The survey does not confirm this correlation, but it does reveal a moderate correlation between how far the conditions for success and risk factors for attaining the desired outcomes are identified and monitored during the implementation phase, and whether RBM is supported by adequate IT systems that are able to process qualitative and quantitative results information.

Figure 4.2. Correlations between key elements of Guiding Principle 2 and Principles 4, 5, and 6

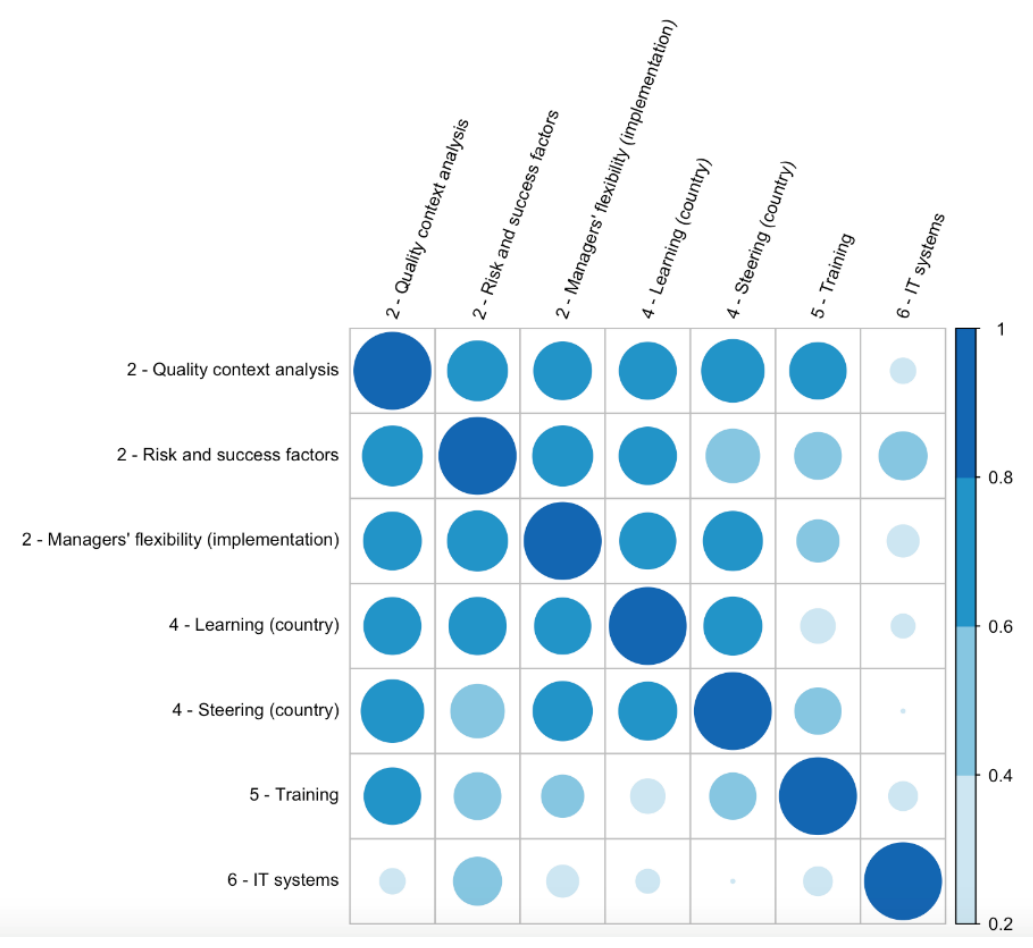

Note: Correlation coefficient used is Spearman's Rho, where values of 0.6 and above indicate a strong relationship.

\section{Correlations between Guiding Principle 3 and key elements of Principles 4, 5 and 6}

Responses to the survey reveal some correlations with Guiding Principles 4 and 5 but nothing significant with Guiding Principle 6.

There is a strong correlation between the quality and transparency of results information and its use. According to Figure 4.3, responses to the survey show a strong correlation between whether or not resultsframeworks, information and data are of a high quality, timely, and made publicly available and whether or not results information is used for learning and decision making at all levels (Guiding Principle 4).

The quality and transparency of results information depends on how leadership is engaging with it. There is a moderate correlation between the extent to which results frameworks, information and data are of a high quality, timely, and made publicly available and if leadership promotes a culture of results and learning by providing guidance, incentives and resources (Guiding Principle 5). 


\section{Correlations between the core components of Guiding Principles 4, 5, and 6}

Figure 4.2 also reveals some significant correlations between Guiding Principles 4, 5 and 6

There is a strong correlation between the use of results information and how far leadership is engaging. There is a strong correlation between how far the results information is used for learning and decision making at all levels (Guiding Principle 4) and if: i) leadership communicates the purpose of RBM and uses results information for programme management and policy influencing; and if ii) leadership promotes a culture of results and learning by providing guidance, incentives, and resources (Guiding Principle 5).

There is a correlation between the use of results information for accountability and the quality of (M\&E) systems. Figure 4.3 also shows a moderate correlation between the extent to which results information is used for accountability at headquarters, and whether or not M\&E systems are set up to produce credible quantitative and qualitative data and evidence that meets an organisation's needs as well as its capacity. This echoes the fact that for many organisations, the main driver for setting up RBM systems, has been accountability.

Figure 4.3. Correlations between Guiding Principles 4, 5, and 6

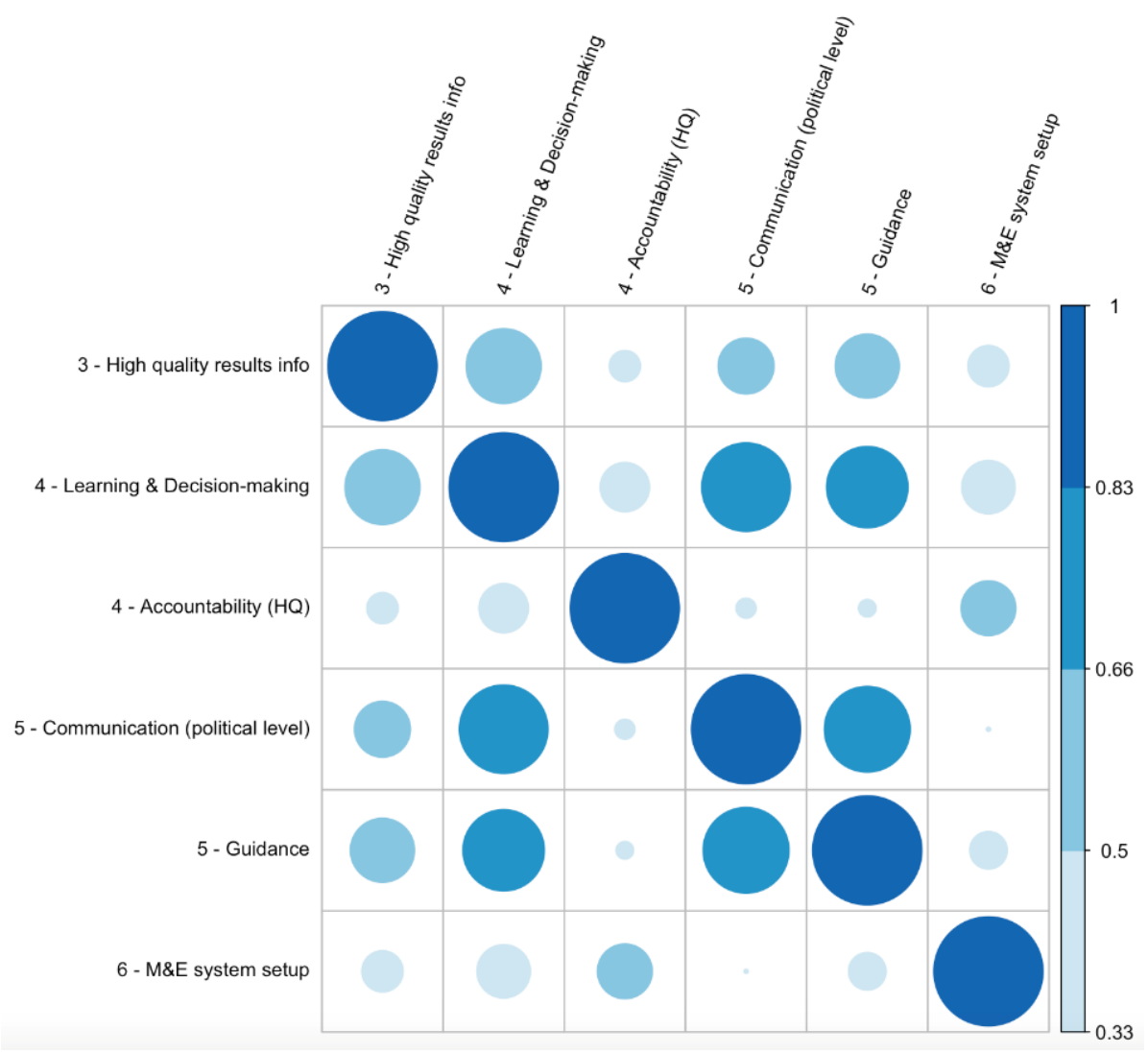

Note: Correlation coefficient used is Spearman's Rho, where values of 0.6 and above indicate a strong relationship. 


\section{Correlations call for holistic approaches in implementing the Guiding Principles}

So far, the correlations above have some limitations and they must be interpreted with caution. These correlations stem from self-assessments, not from objective, external evaluations. Data suggest that organisations with a solid results system would identify stronger correlations It is important to keep in mind that a number of organisations are lagging behind in aligning to Guiding Principles 5 and 6 (chapter 2.1). This might explain for instance, why having a strong RBM system that can generate reliable data (Guiding Principle 6) is only moderately correlated with the use of results information (Guiding Principle 4) and not correlated with Guiding Principles 2 and 3 . We can learn a number of lessons from this.

The use of results information for learning and decision making at all levels depends on all the other Principles. The use of results information is strongly correlated with the extent to which leadership communicates the purpose of results information, how it uses results information, and the ways in which it promotes a culture of results and learning (Guiding Principle 5). It is also correlated with the prevalence of results frameworks at the sector/partner/project level as well as how cross sector approaches are promoted and included into learning and reporting systems of cross-sector approaches (Guiding Principle 1), not to mention the quality and transparency of results information (Guiding Principle 3). There is also a strong correlation between the use of results information at the country level for learning and steering, and how much responsibility managers are given at the field level as well as their focus on achieving long-term outcomes (Guiding Principle 2). There is also a moderate correlation between the use of results information for accountability and the quality of M\&E systems (Guiding Principle 6).

Guiding Principle 5 on building a culture of results and learning conditions the implementation of the other Principles. The role of leadership in promoting a culture of results and learning by communicating the purpose of RBM, using results information, and providing guidance, incentives and resources, including mechanisms for RBM training and peer learning, is crucial. Leadership is also responsible for setting up an appropriate incentive structure, which is one of the constraints indicated by most organisations (chapter 2.2).

\section{Figure 4.4. Correlations between Guiding Principles}

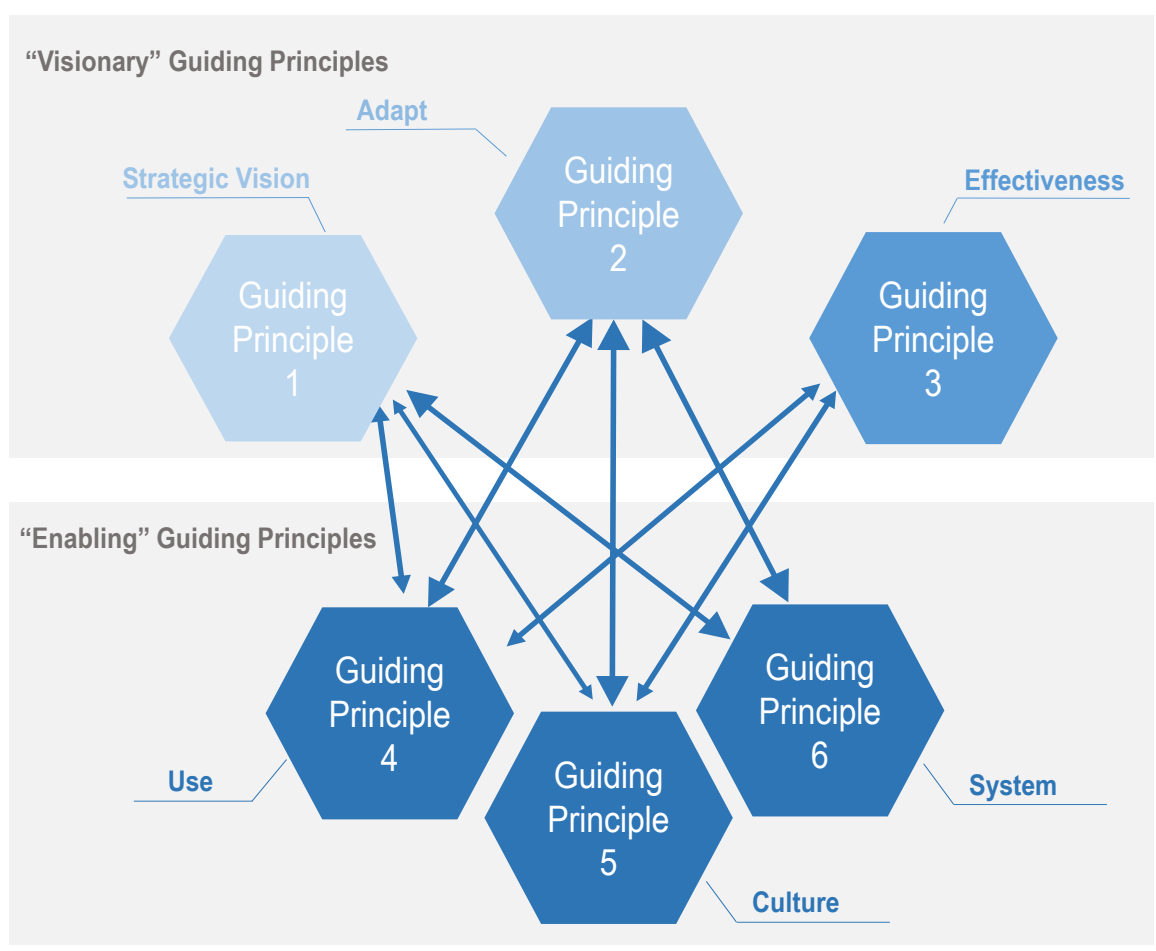




\title{
Practical consequences for
}

\section{systemic and tailored results- based management approaches}

\author{
It is essential to enhance and sustain a broad culture of results and learning as well as adequate \\ information systems
}

It is essential to build a common awareness and a culture of results in order to motivate staff to design results frameworks, to collect data and to use results information. This needs to be accompanied by appropriate incentives and adequate guidelines for staff and partners, with the support of leadership, which also needs to set a good example.

The capacity of individuals and organisations also plays a critical role. Both of them require adequate training to ensure that all stakeholders use the same terminology, based on clear definitions of core RBM terms. They also need to ensure that methodologies for collecting data are applied accordingly, and that results information is also used. This also requires sound and coherent change management processes, focusing on strengthening the capacity of systems used by both providers and partners; allowing for more adaptive management systems; ensuring that there is ample scope for the analysis of results information and peer learning about the successes and the failures. Even in the well-established RBM systems, the incentive structure needs to be looked at more carefully.

Finally, it is also essential to have in place adequate monitoring, evaluation and information systems in order to collect, process, and share data. If these are built in ways that allow users to review their results data, as well as seeing where their data feeds into the whole organisation, they will give both users and producers an even greater incentive to actually collect and use the data.

RBM capacity strengthening needs to be tailored to the profile of each provider. Beyond the attention all organisations need to pay to the organisational and support systems, the three distinct profiles identified by the survey imply different needs, reflecting differences in the degree of maturity of RBM systems. Startuppers who are building different components of their systems will benefit from iterative approaches, where regular stock taking could lead to lessons learned and best practices to incrementally improve the system. Meanwhile builders that are bolstering their RBM systems can focus on specific aspects where they lag behind in aligning to the Guiding Principles, for instance in promoting cross-sector approaches, developing thematic results frameworks, or increasing transparency through systematically publishing their results frameworks and data. Front-runners can lead in developing innovative approaches to accountability and demonstrating results, such as data systems to address complexity and agile corporate results reporting in response to evolving situations happening either at the local or global level such as the COVID-19 pandemic.

The correlations that do exist between the Guiding Principles urge organisations to look at them more holistically as they build their RBM systems. In particular, using results information for learning and 
decision-making at all levels depends on all the other principles. Since organisations are governed under different circumstances and operate in various environments, there is no one-size-fits-all formula. However, there are essential building blocks for RBM systems that all organisations need to have in place. In particular these are: a clear sense of purpose for RBM, theory(ies) of change, results frameworks, monitoring and evaluation systems, documented feedback loops and related reporting and decision making processes, knowledge management and learning systems, as well as incentives for staff to implement RBM approaches (Vähämäki and Verger, 2019[3]).

Providers will need to manage tensions between key elements of the Guiding Principles. As they build on synergies between these Principles, organisations will also need to strike a balance between the key elements of different Principles. For example, having a CRF may contradict the need to align results frameworks to partner country results frameworks. Organisations will also need to clarify how they will use results information for the purpose of steering, learning, communication and accountability.

Using the SDGs can help resolve tensions. As they support partner country ownership while aligning to the SDGs, development co-operation providers will need to decide how to strike a balance. This is between imposing standardised indicators to get aggregate results information at the corporate level, and leaving space for context-based indicators that are aligned to the results frameworks of partner countries. The SDG targets and indicators can potentially help solve this tension. In fact, the survey reveals that extensive use of results-frameworks is correlated with a greater use of SDG indicators: organisations that use resultsframeworks extensively are also more likely to use SDG indicators (Figure C.1, Annex C). Since providers still make limited use of integrated approaches in development co-operation, the SDGs can help organisations move towards more coherent, cross-sector and cross-disciplinary approaches, thereby incorporating those into measurement, learning and reporting systems. Using the SDG framework can also be useful during a crisis where a holistic response is required (OECD, 2020[4]).

Providing appropriate guidance and tools as well as measuring progress overtime can help providers to align to the Guiding Principles. The Results Secretariat will develop a guidance toolkit, using: this survey, the findings from a research on how to make better use of the SDGs as a common framework for results, and the work undertaken with the OECD/DAC Results Community to collect good practices. The good practices and guidance toolkit will be made available on a learning platform with a view to provide the tools that can help members of the OECD/DAC Results Community to implement the Guiding Principles. Peer learning among members through working groups and workshops will continue to be another venue for progress. Finally, this survey will be repeated on a regular basis. Results of the survey will give an objective basis for providers to assess their own progress over time, thereby enabling them to direct their efforts in the areas where they lag behind, while also assessing the overall progress at an aggregate level. 


\section{References}

OECD (2020), Managing for sustainable results in development co-operation in uncertain times, OECD Publishing Paris, https://doi.org/10.1787/44a288bc-en.

OECD (2019), Managing for Sustainable Development Results: Guiding Principles, Best Practices in Development Co-operation, OECD Publishing, Paris, https://dx.doi.org/10.1787/44a288bcen.

OECD (forthcoming), Aligning to the SDGs: Stepping up action, OECD Publishing, Paris.

Vähämäki, J. and C. Verger (2019), "Learning from Results-Based Management evaluations and reviews", OECD Development Co-operation Working Papers, No. 53, OECD Publishing, Paris, https://dx.doi.org/10.1787/3fda0081-en.

\section{Notes}

1 Though the target group and the questions were slightly different, this survey can be compared to an informal survey conducted in 2013. This online survey targeted results focal points in DAC member's headquarters. With responses from 28 out of 35 development ministries and agencies, it provided a snapshot of where DAC members and observers stood on their approach to results.

${ }^{2}$ The detailed list of survey questions can be found in Annex B.

${ }^{3}$ The level of alignment was determined by creating a standardised index (ranging between zero and one) for each Guiding Principle. Given the unequal number of survey questions for each Principle, in order to ensure that these Principles were comparable, it was essential to standardise them. An index was created for each Guiding Principle by adding responses to each question and dividing each respondent's overall score by the number of questions relating to that respective Principle. Since the choice of answers in the survey range from 1 to 4 for all questions related to the Guiding Principles, the choice of answers were rescaled to between 0 and 1 . If for instance, a respondent scored a 1, the lowest score, this was turned into a 0 , a score of 2 was turned into a 0.33 and so on. This was done for each question and then these standardised scores were added up and divided by the number of questions within the principle in order to produce an index, which made it possible to compare respondent's rankings across all Guiding Principles. From these indices a profile for each respondent across all six Guiding Principles can be derived. 
${ }^{4}$ Correlations were calculated as follows: Comparing all responses to all survey questions with each other, Spearman's rho as a correlation coefficient measures the strength of association between two variables, in this case, between how strongly a pair of responses to the survey questions are related. This allows to establish interdependencies between different elements of the Guiding Principles that become apparent in the original survey data. For visibility purposes, only correlations with a coefficient stronger than 0.6 are displayed. All other responses were not systematically correlated with each other. 


\section{Annex A. Survey respondents}

Table A.1. List of survey respondents

\begin{tabular}{|c|c|}
\hline Bilateral providers - Group A & Bilateral providers - Group B \\
\hline Australia & Czech Republic \\
\hline Austria & Estonia \\
\hline Belgium (MFA) & Iceland \\
\hline Belgium (Enabel) & Israel \\
\hline Canada & Latvia \\
\hline Denmark & Lithuania \\
\hline European Union & Mexico \\
\hline Finland & Poland \\
\hline France & Slovak Republic \\
\hline Germany (BMZ) & Slovenia \\
\hline Greece & Multilateral development banks \\
\hline Ireland & African Development Bank \\
\hline Italy & Asian Development Bank \\
\hline Korea & European Bank for Reconstruction and Development \\
\hline Luxembourg (LuxDev) & Inter-American Development Bank \\
\hline Luxembourg (MFA) & Islamic Development Bank \\
\hline Netherlands & World Bank Group \\
\hline \multicolumn{2}{|l|}{ New Zealand } \\
\hline Norway & UN agencies \\
\hline Portugal & Food and Agriculture Organisation \\
\hline Spain & United Nations Development Programme \\
\hline Sweden (MFA) & United Nations Educational, Scientific and Cultural Organization \\
\hline Sweden (SIDA) & United Nations Children's Fund \\
\hline Switzerland (SDC) & United Nations Industrial Development Organization \\
\hline Switzerland (SECO) & World Food Programme \\
\hline \multicolumn{2}{|l|}{ United Kingdom } \\
\hline United States of America & \\
\hline
\end{tabular}




\title{
Annex B. Survey questions
}

\author{
Table B.1. List of survey questions
}

\begin{tabular}{|c|c|c|}
\hline $\begin{array}{l}\text { Guiding } \\
\text { Principle }\end{array}$ & & $\begin{array}{l}\text { Numerica } \\
\text { code }\end{array}$ \\
\hline 1 & Does your organisation have a corporate results framework in place? & Q111 \\
\hline 1 & Results frameworks are developed at the following levels: & \\
\hline 1 & At sector/thematic level & Q121 \\
\hline 1 & At partner country level & Q122 \\
\hline 1 & At project level & Q123 \\
\hline 1 & To what extent do your results frameworks incorporate SDG results indicators? & \\
\hline 1 & At corporate level & Q131 \\
\hline 1 & At sector/thematic level & Q132 \\
\hline 1 & At partner country level & Q133 \\
\hline 1 & At project level & Q134 \\
\hline 1 & $\begin{array}{l}\text { Established methodologies (e.g. logical framework, theory of change) are used to define the results chain at the following } \\
\text { levels. }\end{array}$ & \\
\hline 1 & At corporate level & Q141 \\
\hline 1 & At sector/thematic level & Q142 \\
\hline 1 & At partner country level & Q143 \\
\hline 1 & At project level & Q144 \\
\hline 1 & Results mainstreaming and coherence. & \\
\hline 1 & The design of the organisational structure is geared towards achieving development results. & Q151 \\
\hline 1 & $\begin{array}{l}\text { In line with the integrated approach of the SDGs, cross-sector approaches are promoted and included in measurement, } \\
\text { learning and reporting systems. }\end{array}$ & Q152 \\
\hline 2 & $\begin{array}{l}\text { Thinking about your development co-operation portfolio in partner countries, which of the following practices are you } \\
\text { adopting in order to adapt to context and be flexible? }\end{array}$ & \\
\hline 2 & $\begin{array}{l}\text { Quality context analysis enabling a sound understanding of local dynamics and needs inform country- and project-level } \\
\text { results frameworks. }\end{array}$ & Q21 \\
\hline 2 & $\begin{array}{l}\text { The conditions for success (and/or assumptions) and risk factors in achieving the intended outcomes are identified and } \\
\text { are monitored during implementation. }\end{array}$ & Q22 \\
\hline 2 & $\begin{array}{l}\text { Managers at country level have sufficient flexibility to tailor their approaches and processes to each specific context } \\
\text { (design phase). }\end{array}$ & Q23 \\
\hline 2 & $\begin{array}{l}\text { Managers at country level focus on achieving long-term outcomes, and are empowered to adjust the path of a project in } \\
\text { light of changing local contexts and results information to ensure the expected outcomes are met (implementation } \\
\text { phase). }\end{array}$ & Q24 \\
\hline 3 & To enhance country ownership, mutual accountability and transparency: & \\
\hline 3 & Country-level results frameworks are aligned with partner countries' results frameworks, including at indicator level. & Q31 \\
\hline 3 & The organisation's planning cycles at country level are synchronised with partner country planning cycles. & Q32 \\
\hline 3 & $\begin{array}{l}\text { Country and project level results frameworks are developed in a participatory way, and inclusive involvement is sought in } \\
\text { problem analysis, design, planning, monitoring, evaluation and in the use of results. }\end{array}$ & Q33 \\
\hline 3 & Monitoring approaches maximise the use of partner countries' monitoring and statistical systems. & Q34 \\
\hline 3 & Efforts are made to strengthen countries' statistical and monitoring capacities where needed. & Q35 \\
\hline 3 & Mutual accountability is actively pursued at country- and project-level with regular dialogue among partners. & Q36 \\
\hline 3 & Results frameworks, information and data are high quality, timely, and publicly available. & Q37 \\
\hline 4 & In maximising the use of results information for learning and decision-making. & \\
\hline 4 & Results information from M\&E is used for learning and decision-making at all levels. & Q41 \\
\hline 4 & Qualitative information is collected to complement quantitative data. & Q42 \\
\hline
\end{tabular}




\begin{tabular}{|c|c|c|}
\hline 4 & The purpose and use of results information are clearly specified to staff and partners. & Q43 \\
\hline 4 & At headquarters level, results information is used for: & \\
\hline 4 & Accountability & Q441 \\
\hline 4 & Communicating & Q442 \\
\hline 4 & Learning & Q443 \\
\hline 4 & Steering/Decision-making & Q444 \\
\hline 4 & At country-level, results information is used for: & \\
\hline 4 & Accountability & Q451 \\
\hline 4 & Communicating & Q452 \\
\hline 4 & Learning & Q453 \\
\hline 4 & Steering/Decision-making & Q454 \\
\hline 5 & To foster a culture of results and learning & \\
\hline 5 & $\begin{array}{r}\text { Leadership communicates the purpose of RBM and uses results information for overall programme management and } \\
\text { policy influencing at a political level }\end{array}$ & Q51 \\
\hline 5 & $\begin{array}{r}\text { Leadership promotes a culture of results and learning by providing appropriate guidance, tailored incentives, and } \\
\text { resources for managing for sustainable development results }\end{array}$ & Q52 \\
\hline 5 & All relevant staff are trained in RBM approaches and methods. & Q53 \\
\hline 5 & There are room and mechanisms for peer learning on successes and failures & Q54 \\
\hline 5 & The organisation values careful experimentation in support of innovation as a way to foster better results. & Q55 \\
\hline 6 & To develop a results system that is manageable and reliable. & \\
\hline 6 & Monitoring and evaluation systems are adequately resourced to meet the needs of the organisation. & Q61 \\
\hline 6 & $\begin{array}{l}\text { Monitoring and evaluation systems are set up to produce credible quantitative and qualitative data and evidence that } \\
\text { meets the needs and capacities of the development organisation and its partners. }\end{array}$ & Q62 \\
\hline 6 & $\begin{array}{l}\text { Results based management is supported by adequate information systems, able to manage quantitative and qualitative } \\
\text { results information. }\end{array}$ & Q63 \\
\hline 6 & The overall RBM system is user friendly, lean, and reviewed regularly to fit its purpose. & Q64 \\
\hline $\begin{array}{l}\text { Strengths } \\
\text { and } \\
\text { constraints }\end{array}$ & $\begin{array}{l}\text { In reflecting upon the strengths and constraints for developing your RBM system further, where do you situate your } \\
\text { organisation in the following areas? } \\
\text { Policy/Policies. } \\
\text { Organisational structure. } \\
\text { Internal organisational culture. } \\
\text { Dedicated resources. } \\
\text { Adequate skills \& training. } \\
\text { Internal processes. } \\
\text { Incentive structure. } \\
\text { Quality of results methodologies. } \\
\text { Quality assurance mechanisms. } \\
\text { Information technology systems (IT). } \\
\text { Formal demand for results information reporting. } \\
\text { Actual use of results information. } \\
\text { To be rated from } 1 \text { "Major Constraint" to } 10 \text { "Major Strength" }\end{array}$ & \\
\hline
\end{tabular}




\section{Annex C. Strengths and constraints - Detailed results}

Table C.1. Assessment of strengths and constraints

Aggregated averages per respondents' category $(\mathrm{N}=50)$

\begin{tabular}{|c|c|c|c|c|c|}
\hline & Overall & Bilaterals / Group A & Bilaterals / Group B & $\begin{array}{c}\text { Multilateral } \\
\text { Development Banks }\end{array}$ & UN Agencies \\
\hline Policies & 6.83 & 7.59 & 5.45 & 7.6 & 6.83 \\
\hline $\begin{array}{l}\text { Organisational } \\
\text { structures }\end{array}$ & 5.75 & 6.03 & 4.45 & 7.2 & 5.66 \\
\hline $\begin{array}{l}\text { Internal } \\
\text { organisational culture }\end{array}$ & 5.26 & 5.29 & 5.18 & 5.5 & 5 \\
\hline Dedicated resources & 4.89 & 5.03 & 3.81 & 6.4 & 5 \\
\hline $\begin{array}{l}\text { Adequate skills and } \\
\text { training }\end{array}$ & 5.3 & 5.37 & 4.72 & 6.16 & 5.16 \\
\hline Internal processes & 5.68 & 5.96 & 4.54 & 6.66 & 5.5 \\
\hline Incentive structure & 4.44 & 4.18 & 5.4 & 4 & 4 \\
\hline $\begin{array}{l}\text { Quality of results } \\
\text { methodologies }\end{array}$ & 6.26 & 6.85 & 4.45 & 7.2 & 6.16 \\
\hline $\begin{array}{l}\text { Quality assurance } \\
\text { mechanisms }\end{array}$ & 6 & 6.44 & 4.81 & 7 & 5.16 \\
\hline $\begin{array}{l}\text { Information } \\
\text { technology (IT) } \\
\text { systems }\end{array}$ & 4.95 & 4.96 & 4.72 & 5.6 & 4.83 \\
\hline $\begin{array}{l}\text { Formal demand for } \\
\text { results information } \\
\text { reporting }\end{array}$ & 6.77 & 7.14 & 5.54 & 7.8 & 6.5 \\
\hline $\begin{array}{l}\text { Actual use of results } \\
\text { information }\end{array}$ & 5.74 & 6.07 & 4.90 & 6.66 & 4.83 \\
\hline
\end{tabular}

Note: Answer choices ranking from 1 (Major weakness) to 10 (Major strength), where $7+$ is considered good enough and 4 and below is considered insufficient.

Table C.2. Aggregate responses per Guiding Principle and best performers' scores

\begin{tabular}{l|r|r|r}
\hline & & Average response & 75 $^{\text {th }}$ percentile \\
\hline \multirow{3}{*}{ GP 1 } & Q151 & 2.55 & 3 \\
\hline \multirow{3}{*}{ GP 2 } & Q152 & 2.32 & 3 \\
\cline { 2 - 4 } & Q21 & 3.14 & 4 \\
\cline { 2 - 4 } & Q22 & 2.87 & 4 \\
\cline { 2 - 4 } & Q23 & 3.12 & 4 \\
\hline \multirow{5}{*}{ GP 3 } & Q24 & 2.71 & 3 \\
\cline { 2 - 4 } & Q31 & 2.30 & 3 \\
\cline { 2 - 4 } & Q32 & 2.26 & 3 \\
\cline { 2 - 4 } & Q33 & 2.66 & 3 \\
\hline
\end{tabular}




\begin{tabular}{|c|c|c|c|}
\hline & Q35 & 2.24 & 3 \\
\hline & Q36 & 2.78 & 4 \\
\hline & Q37 & 2.26 & 3 \\
\hline \multirow[t]{3}{*}{ GP 4} & Q41 & 2.36 & 3 \\
\hline & Q42 & 2.74 & 3 \\
\hline & Q43 & 2.58 & 3 \\
\hline \multirow[t]{5}{*}{ GP 5} & Q51 & 2.32 & 3 \\
\hline & Q52 & 2.22 & 3 \\
\hline & Q53 & 2.3 & 3 \\
\hline & Q54 & 2.4 & 3 \\
\hline & Q55 & 2.32 & 3 \\
\hline \multirow[t]{4}{*}{ GP 6} & Q611 & 2.34 & 3 \\
\hline & Q612 & 2.52 & 3 \\
\hline & Q613 & 2.12 & 3 \\
\hline & Q614 & 2.16 & 3 \\
\hline
\end{tabular}

Figure C.1. To what extent are Sustainable Development Goals indicators used in providers' results frameworks?
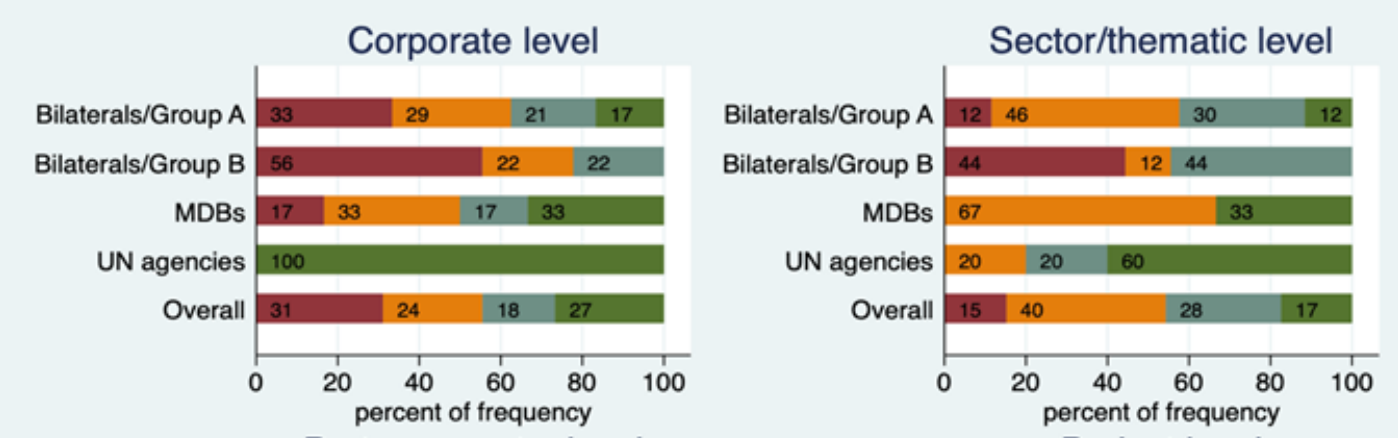

Partner country level
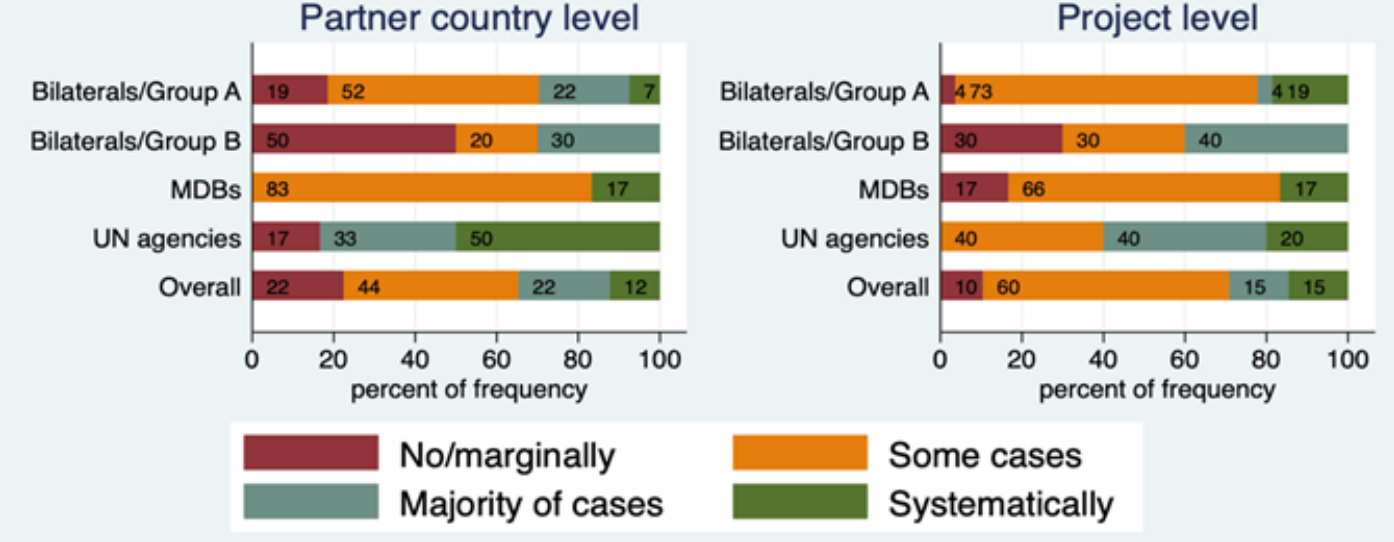
Figure C.2. Extent to which providers use established methodologies to develop their results frameworks (at the following levels)

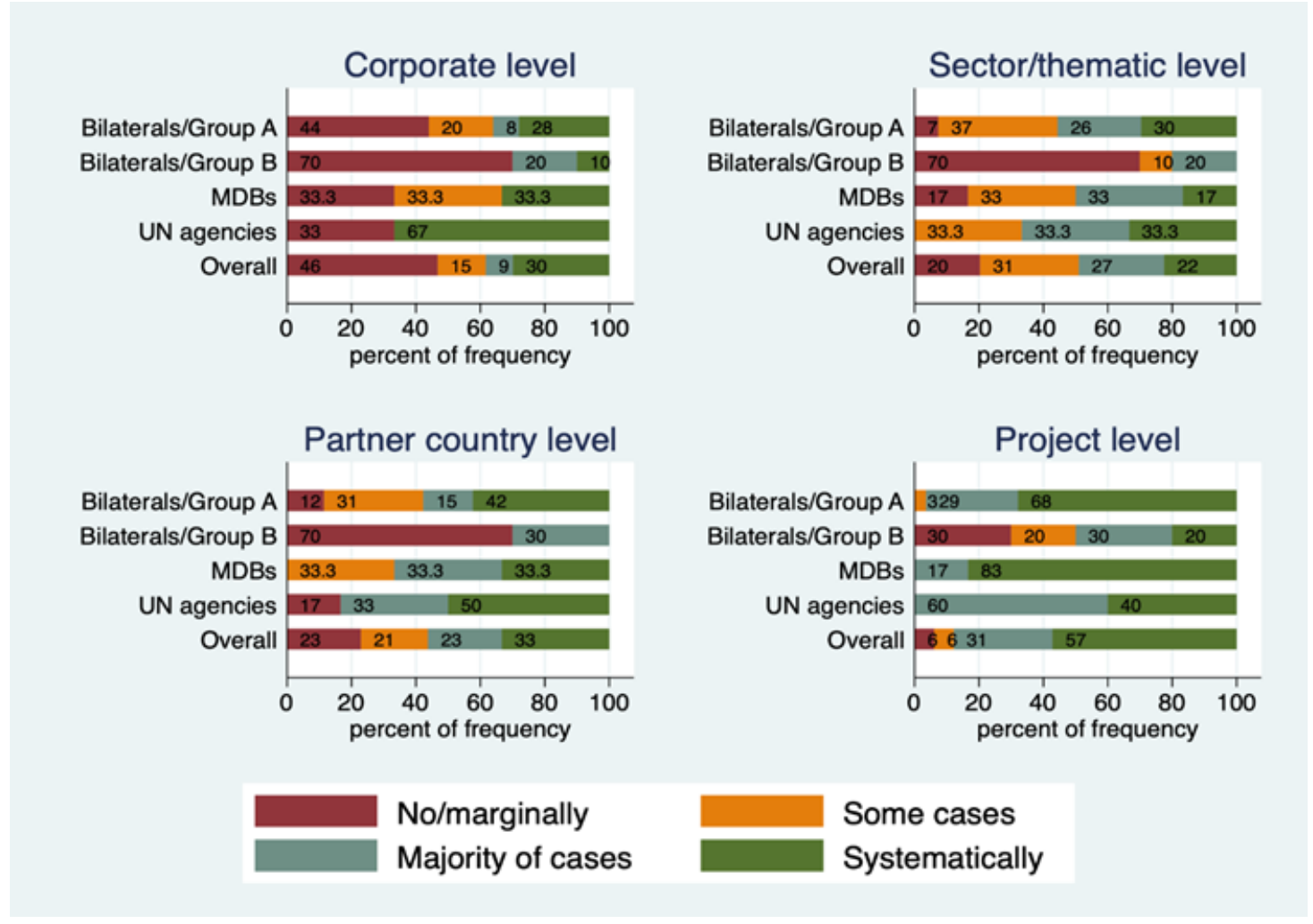

Figure C.3. Results frameworks and Sustainable Development Goals indicators

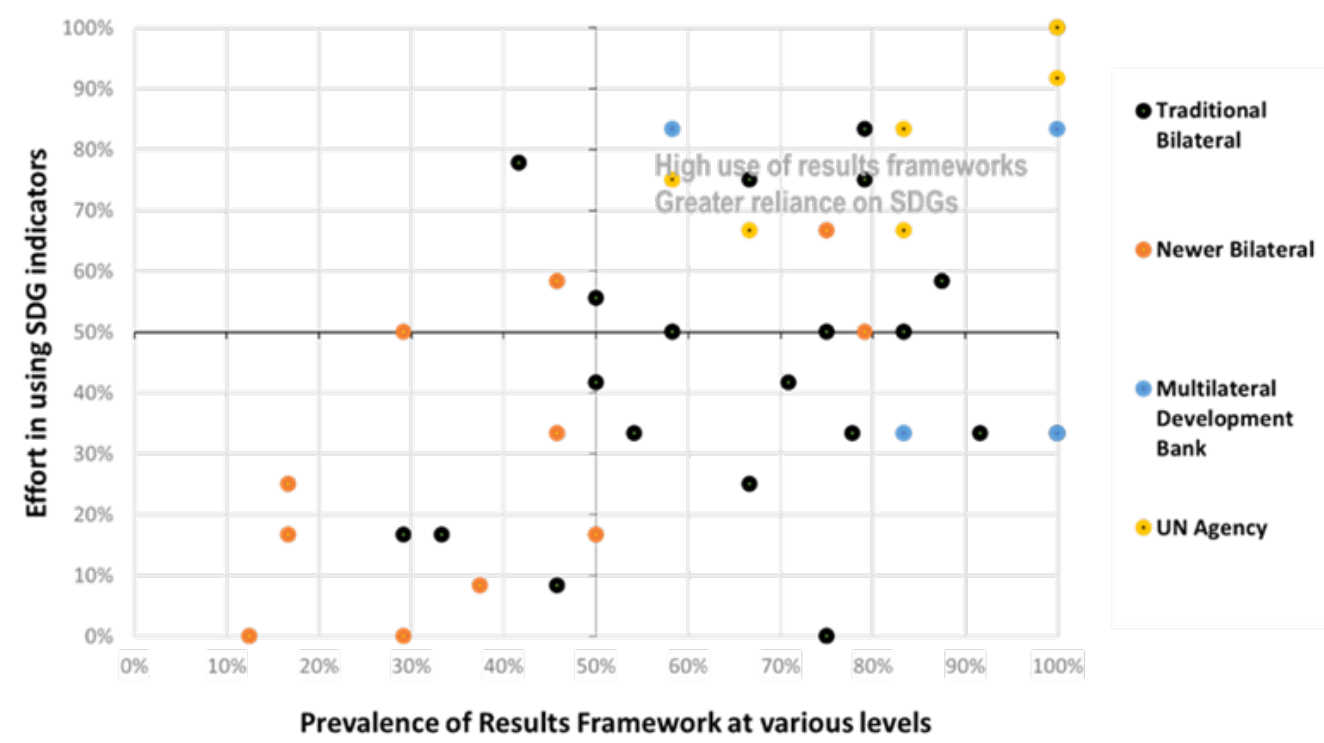

